\title{
BOUNDARY VALUE PROBLEMS FOR DEGENERATE AND DEGENERATE FRACTIONAL ORDER DIFFERENTIAL EQUATIONS WITH NON-LOCAL LINEAR SOURCE AND DIFFERENCE METHODS FOR THEIR NUMERICAL IMPLEMENTATION
}

\author{
M.Kh. BESHTOKOV
}

\begin{abstract}
In the paper we study non-local boundary value problems for differential and partial differential equations of fractional order with a non-local linear source being mathematical models of the transfer of water and salts in soils with fractal organization. Apart of the Cartesian case, in the paper we consider one-dimensional cases with cylindrical and spherical symmetry. By the method of energy inequalities, we obtain apriori estimates of solutions to nonlocal boundary value problems in differential form. We construct difference schemes and for these schemes, we prove analogues of apriori estimates in the difference form and provide estimates for errors assuming a sufficient smoothness of solutions to the equations. By the obtained apriori estimates, we get the uniqueness and stability of the solution with respect to the the initial data and the right par, as well as the convergence of the solution of the difference problem to the solution of the corresponding differential problem with the rate of $O\left(h^{2}+\tau^{2}\right)$.
\end{abstract}

Keywords: boundary value problem, apriori estimate, the equation of moisture transfer, the differential equation of fractional order, Gerasimov-Caputo fractional derivative.

Mathematics Subject Classification: 65N06; 65N12

\section{INTRODUCTION}

As non-local boundary value problems, one usually calls problem, in which on the boundary, instead of prescribing the values of a solution and its derivatives, a relation between these values and similar values on some other internal or boundary manifolds is given. The theory of non-local boundary value problems is important as a part of general theory of boundary value problems for partial differential equations due to numerous applications in mechanics, physics, biology and other natural sciences.

At present, differential equations involving fractional derivatives both in time and spatial variables attract attention by mathematicians and physicians since such equations serve as mathematical models of various processes [1]-9]. A lot of works are devoted to studying various local and non-local initial-boundary value problems for Sobolev type differential equations and their subclass of pseudo-parabolic equations [10]-[19]. In works [20]-[25], the finite difference method was employed for studying various boundary value problems for Sobolev type differential equations with varying coefficients.

M.Kh. Beshtokov, Boundary value problems for Degenerate and Degenerate fractional ORDER DIFFERENTIAL EQUATIONS WITH NON-LOCAL LINEAR SOURCE AND DIFFERENCE METHODS FOR THEIR NUMERICAL IMPLEMENTATION.

(C)Beshtokov M.KH. 2019.

Submitted May 29, 2018. 
In the present work we consider nonlocal boundary value problems for Sobolev type differential equations with a Gerasimov-Caputo fractional derivative in time and with a nonlocal linear source. Apart of the Cartesian case, in the work we consider one-dimensional cases with a cylindrical and spherical symmetry.

\section{Boundary VAlue PROBlem For a PSEUdo-PARABOliC EQUATION With A NONLOCAL LINEAR SOURCE}

In a closed cylinder $\bar{Q}_{T}=\{(x, t): 0 \leqslant x \leqslant l, 0 \leqslant t \leqslant T\}$ we consider the following boundary value problem:

$$
\begin{aligned}
\partial_{0 t}^{\alpha} u= & \frac{\partial}{\partial x}\left(k(x, t) \frac{\partial u}{\partial x}\right)+\partial_{0 t}^{\alpha} \frac{\partial}{\partial x}\left(\eta(x) \frac{\partial u}{\partial x}\right)+r(x, t) \frac{\partial u}{\partial x} \\
& -\int_{0}^{x} q(s, t) u(s, t) d s+f(x, t), \quad 0<x<l, \quad 0<t \leqslant T, \\
\Pi(0, t) & =\beta_{11}(t) u(0, t)+\beta_{12}(t) \partial_{0 t}^{\alpha} u(0, t)-\mu_{1}(t), \quad 0 \leqslant t \leqslant T, \\
-\Pi(l, t) & =\beta_{21}(t) u(l, t)+\beta_{22}(t) \partial_{0 t}^{\alpha} u(l, t)-\mu_{2}(t), \quad 0 \leqslant t \leqslant T, \\
u(x, 0) & =u_{0}(x), \quad 0 \leqslant x \leqslant l,
\end{aligned}
$$

where

$$
\begin{aligned}
& 0<c_{0} \leqslant k(x, t), \eta(x), \quad \beta_{12}(t), \quad \beta_{22}(t) \leqslant c_{1}, \\
& \left|\beta_{11}(t), \beta_{21}(t), r(x, t), q(x, t), \eta_{x}(x), k_{x}(x, t), r_{x}(x, t)\right| \leqslant c_{2},
\end{aligned}
$$

and

$$
\partial_{0 t}^{\alpha} u=\frac{1}{\Gamma(1-\alpha)} \int_{0}^{t} \frac{u_{\tau}(x, \tau)}{(t-\tau)^{\alpha}} d \tau
$$

is a Gerasimov-Caputo fractional derivative of order $\alpha, 0<\alpha<1$, [26], [27], $\Pi(x, t)=$ $k u_{x}+\partial_{0 t}^{\alpha}\left(\eta(x) u_{x}\right), c_{i}, i=0,1,2$ are positive constants,

$$
\partial_{0 t}^{\alpha} u=D_{0 t}^{\alpha} u-\frac{u(0)}{\Gamma(1-\alpha) t^{\alpha}}, \quad D_{0 t}^{\alpha} u=\frac{1}{\Gamma(1-\alpha)} \frac{d}{d t} \int_{0}^{t} \frac{u d \tau}{(t-\tau)^{\alpha}}
$$

is the Riemann-Liouville fractional derivative of order $\alpha$.

In what follows we assume that problem (1.1)-(1.4) possesses the unique solutions with all needed derivatives. We shall assume that the coefficients in the equation and boundary conditions satisfy all smoothness conditions arising in calculations and ensuring a needed approximation order of the difference scheme. By $M_{i}, i=1,2, \ldots$, we shall denote positive constants depending only on the data of the considered problem.

\section{APRIORI ESTIMATE IN DIFFERENTIAL FORM}

To obtain apriori estimate for the solution of problem (1.1)-(1.4) in a a differential form, we introduce a scalar product and a norm:

$$
(a, b)=\int_{0}^{l} a b d x, \quad(a, a)=\|a\|_{0}^{2},
$$

where $a, b$ are some given on $[0, l]$ functions. We multiply equation (1.1) by $U=u+\partial_{0 t}^{\alpha} u$ in the sense of the scalar product:

$$
\left(\partial_{0 t}^{\alpha} u, U\right)=\left(\left(k u_{x}\right)_{x}, U\right)+\left(\partial_{0 t}^{\alpha}\left(\eta u_{x}\right)_{x}, U\right)+\left(r u_{x}, U\right)-\left(\int_{0}^{x} q u d s, U\right)+(f, U) .
$$

The following lemma holds true [28]. 
Lemma 1. For each absolutely continuous on $[0, T]$ function $v(t)$, the inequality holds:

$$
v(t) \partial_{0 t}^{\alpha} v(t) \geqslant \frac{1}{2} \partial_{0 t}^{\alpha}\left(v^{2}(t)\right), \quad 0<\alpha<1
$$

We rewrite the terms in identity (2.1) by employing the Cauchy inequality with $\varepsilon$ [29] and Lemma 1:

$$
\begin{aligned}
& \left(\partial_{0 t}^{\alpha} u, U\right)=\left(\partial_{0 t}^{\alpha} u, u+\partial_{0 t}^{\alpha} u\right)=\left(1, u \partial_{0 t}^{\alpha} u\right)+\left(1,\left(\partial_{0 t}^{\alpha} u\right)^{2}\right) \geqslant \frac{1}{2} \partial_{0 t}^{\alpha}\|u\|_{0}^{2}+\left\|\partial_{0 t}^{\alpha} u\right\|_{0}^{2}, \\
& \left(\left(k u_{x}\right)_{x}, U\right)=\left(\left(k u_{x}\right)_{x}, u+\partial_{0 t}^{\alpha} u\right)=\left.U k u_{x}\right|_{0} ^{l}-\left(k u_{x}, u_{x}+\partial_{0 t}^{\alpha} u_{x}\right) \\
& =\left.U k u_{x}\right|_{0} ^{l}-\left(k, u_{x}^{2}\right)-\left(k, u_{x} \partial_{0 t}^{\alpha} u_{x}\right) \\
& \leqslant\left. U k u_{x}\right|_{0} ^{l}-c_{0}\left\|u_{x}\right\|_{0}^{2}-\frac{1}{2} \int_{0}^{l} k \partial_{0 t}^{\alpha}\left(u_{x}\right)^{2} d x \text {. } \\
& \left(\partial_{0 t}^{\alpha}\left(\eta u_{x}\right)_{x}, U\right)=\left(\partial_{0 t}^{\alpha}\left(\eta u_{x}\right)_{x}, u+\partial_{0 t}^{\alpha} u\right)=\left.U \partial_{0 t}^{\alpha}\left(\eta u_{x}\right)\right|_{0} ^{l}-\left(\partial_{0 t}^{\alpha}\left(\eta u_{x}\right), u_{x}+\partial_{0 t}^{\alpha} u_{x}\right) \\
& =-\left(\eta, u_{x} \partial_{0 t}^{\alpha} u_{x}\right)-\left(\eta,\left(\partial_{0 t}^{\alpha} u_{x}\right)^{2}\right)+\left.U \partial_{0 t}^{\alpha}\left(\eta u_{x}\right)\right|_{0} ^{l} \\
& =\left.U \partial_{0 t}^{\alpha}\left(\eta u_{x}\right)\right|_{0} ^{l}-\frac{1}{2} \int_{0}^{l} \eta(x) \partial_{0 t}^{\alpha}\left(u_{x}\right)^{2} d x-c_{0}\left\|\partial_{0 t}^{\alpha} u_{x}\right\|_{0}^{2} \text {. } \\
& \left(r u_{x}, U\right)=\left(r u_{x}, u+\partial_{0 t}^{\alpha} u\right)=\left(r u_{x}, u\right)+\left(r u_{x}, \partial_{0 t}^{\alpha} u\right) \\
& \leqslant \varepsilon\left\|\partial_{0 t}^{\alpha} u\right\|_{0}^{2}+M_{1}^{\varepsilon}\left(\|u\|_{0}^{2}+\left\|u_{x}\right\|_{0}^{2}\right), \\
& -\left(\int_{0}^{s} q u d s, U\right)=-\left(\int_{0}^{s} q u d s, u+\partial_{0 t}^{\alpha} u\right)=-\left(\int_{0}^{s} q u d s, u\right)-\left(\int_{0}^{s} q u d s, \partial_{0 t}^{\alpha} u\right) \\
& \leqslant \varepsilon\left\|\partial_{0 t}^{\alpha} u\right\|_{0}^{2}+\frac{1}{2}\|u\|_{0}^{2}+M_{2}^{\varepsilon}\left(1,\left(\int_{0}^{s} q u d s\right)^{2}\right) \\
& \leqslant M_{3}^{\varepsilon} \int_{0}^{l} \int_{0}^{s} u^{2} d s d x+\varepsilon\left\|\partial_{0 t}^{\alpha} u\right\|_{0}^{2}+\frac{1}{2}\|u\|_{0}^{2} \leqslant \varepsilon\left\|\partial_{0 t}^{\alpha} u\right\|_{0}^{2}+M_{4}^{\varepsilon}\|u\|_{0}^{2} . \\
& (f, U)=\left(f, u+\partial_{0 t}^{\alpha} u\right)=(f, u)+\left(f, \partial_{0 t}^{\alpha} u\right) \leqslant \varepsilon\left\|\partial_{0 t}^{\alpha} u\right\|_{0}^{2}+M_{5}^{\varepsilon}\|f\|_{0}^{2}+\|u\|_{0}^{2} .
\end{aligned}
$$

Taking into considerations transformations (2.2)-(2.7), by (2.1) we find

$$
\begin{aligned}
\frac{1}{2} \partial_{0 t}^{\alpha}\|u\|_{0}^{2} & +\left\|\partial_{0 t}^{\alpha} u\right\|_{0}^{2}+c_{0}\left\|u_{x}\right\|_{0}^{2}+\frac{1}{2} \int_{0}^{l}(k+\eta(x)) \partial_{0 t}^{\alpha}\left(u_{x}\right)^{2} d x+c_{0}\left\|\partial_{0 t}^{\alpha} u_{x}\right\|_{0}^{2} \\
& \leqslant\left. U \Pi(x, t)\right|_{0} ^{l}+\varepsilon\left\|\partial_{0 t}^{\alpha} u\right\|_{0}^{2}+M_{6}^{\varepsilon}\left(\|u\|_{0}^{2}+\left\|u_{x}\right\|_{0}^{2}\right)+M_{7}^{\varepsilon}\|f\|_{0}^{2}
\end{aligned}
$$

Letting $\varepsilon=\frac{1}{2}$, by $(2.8)$ we get:

$$
\begin{aligned}
\frac{1}{2} \partial_{0 t}^{\alpha}\|u\|_{0}^{2} & +\frac{1}{2}\left\|\partial_{0 t}^{\alpha} u\right\|_{0}^{2}+c_{0}\left\|u_{x}\right\|_{0}^{2}+\frac{1}{2} \int_{0}^{l}(k+\eta(x)) \partial_{0 t}^{\alpha}\left(u_{x}\right)^{2} d x+c_{0}\left\|\partial_{0 t}^{\alpha} u_{x}\right\|_{0}^{2} \\
& \leqslant\left. U \Pi(x, t)\right|_{0} ^{l}+M_{8}\left(\|u\|_{0}^{2}+\left\|u_{x}\right\|_{0}^{2}\right)+M_{9}\|f\|_{0}^{2} .
\end{aligned}
$$


Estimating the first term in the right hand side of the above inequality, we obtain:

$$
\begin{aligned}
\left.U(x, t) \Pi(x, t)\right|_{0} ^{l}= & \left(u(l, t)+\partial_{0 t}^{\alpha} u(l, t)\right)\left(\mu_{2}(t)-\beta_{21}(t) u(l, t)-\beta_{22}(t) \partial_{0 t}^{\alpha} u(l, t)\right) \\
& +\left(u(0, t)+\partial_{0 t}^{\alpha} u(0, t)\right)\left(\mu_{1}(t)-\beta_{11} u(0, t)-\beta_{12}(t) \partial_{0 t}^{\alpha} u(0, t)\right) \\
= & \mu_{2}(t) u(l, t)+\mu_{2}(t) \partial_{0 t}^{\alpha} u(l, t)-\beta_{21}(t) u^{2}(l, t)-\beta_{21}(t) u(l, t) \partial_{0 t}^{\alpha} u(l, t) \\
& -\beta_{22}(t) u(l, t) \partial_{0 t}^{\alpha} u(l, t)-\beta_{22}(t)\left(\partial_{0 t}^{\alpha} u(l, t)\right)^{2}+\mu_{1}(t) u(0, t) \\
& +\mu_{1}(t) \partial_{0 t}^{\alpha} u(0, t)-\beta_{11}(t) u^{2}(0, t)-\beta_{11} u(0, t) \partial_{0 t}^{\alpha} u(0, t) \\
& -\beta_{12}(t) u(0, t) \partial_{0 t}^{\alpha} u(0, t)-\beta_{12}\left(\partial_{0 t}^{\alpha} u(0, t)\right)^{2} \\
\leqslant & M_{10}^{\varepsilon_{1}, \varepsilon_{2}}\left(\mu_{1}^{2}+\mu_{2}^{2}\right)+\varepsilon_{1}\left(\partial_{0 t}^{\alpha} u(l, t)\right)^{2}+\varepsilon_{2}\left(\partial_{0 t}^{\alpha} u(0, t)\right)^{2} \\
& +M_{11}^{\varepsilon}\left(\|u\|_{0}^{2}+\left\|u_{x}\right\|_{0}^{2}\right)-\beta_{22}(t)\left(\partial_{0 t}^{\alpha} u(l, t)\right)^{2}-\frac{1}{2} \beta_{22}(t) \partial_{0 t}^{\alpha} u^{2}(l, t) \\
& -\beta_{12}(t)\left(\partial_{0 t}^{\alpha} u(0, t)\right)^{2}-\frac{1}{2} \beta_{12}(t) \partial_{0 t}^{\alpha} u^{2}(0, t) \\
\leqslant & -\frac{\beta_{12}(t)}{2}\left(\partial_{0 t}^{\alpha} u(0, t)\right)^{2}-\frac{\beta_{22}(t)}{2}\left(\partial_{0 t}^{\alpha} u(l, t)\right)^{2}-\frac{\beta_{12}(t)}{2} \partial_{0 t}^{\alpha} u^{2}(0, t) \\
& -\frac{\beta_{12}(t)}{2} \partial_{0 t}^{\alpha} u^{2}(l, t)+M_{12}\left(\|u\|_{0}^{2}+\left\|u_{x}\right\|_{0}^{2}\right)+M_{13}\left(\mu_{1}^{2}+\mu_{2}^{2}\right) .
\end{aligned}
$$

In view of (2.10), by (2.9) we find:

$$
\begin{aligned}
\partial_{0 t}^{\alpha}\|u\|_{0}^{2} & +\int_{0}^{l}(k+\eta(x)) \partial_{0 t}^{\alpha}\left(u_{x}\right)^{2} d x+\left\|u_{x}\right\|_{0}^{2}+\left\|\partial_{0 t}^{\alpha} u\right\|_{0}^{2}+\left\|\partial_{0 t}^{\alpha} u_{x}\right\|_{0}^{2} \\
& \leqslant M_{14}\|u\|_{W_{2}^{1}(0, l)}^{2}+M_{15}\left(\|f\|_{0}^{2}+\mu_{1}^{2}(t)+\mu_{2}^{2}(t)\right)
\end{aligned}
$$

where $\|u\|_{W_{2}^{1}(0, l)}^{2}=\|u\|_{0}^{2}+\left\|u_{x}\right\|_{0}^{2}$.

We apply the operator of fractional integration $D_{0 t}^{-\alpha}$ to both sides of inequality (2.11) and we obtain:

$$
\begin{aligned}
\|u\|_{W_{2}^{1}(0, l)}^{2} & +D_{0 t}^{-\alpha}\left(\left\|u_{x}\right\|_{0}^{2}+\left\|\partial_{0 t}^{\alpha} u\right\|_{0}^{2}+\left\|\partial_{0 t}^{\alpha} u_{x}\right\|_{0}^{2}\right) \leqslant M_{14} D_{0 t}^{-\alpha}\|u\|_{W_{2}^{1}(0, l)}^{2} \\
& +M_{16}\left(D_{0 t}^{-\alpha}\left(\|f\|_{0}^{2}+\mu_{1}^{2}(t)+\mu_{2}^{2}(t)\right)+\left\|u_{0}(x)\right\|_{W_{2}^{1}(0, l)}^{2}\right) .
\end{aligned}
$$

To estimate the first term in the right hand side, we employ Lemma [28].

Lemma 2. Let a non-negative absolutely continuous function $y(t)$ for almost all $t$ in $[0, T]$ satisfy the inequality

$$
\partial_{0 t}^{\alpha} y(t) \leqslant c_{1} y(t)+c_{2}(t), \quad 0 \leqslant \alpha \leqslant 1
$$

where $c_{1}>0, c_{2}(t)$ is a summable on $[0, T]$ non-negative function. Then

$$
y(t) \leqslant y(0) E_{\alpha}\left(c_{1} t^{\alpha}\right)+\Gamma(\alpha) E_{\alpha, \alpha}\left(c_{1} t^{\alpha}\right) D_{0 t}^{-\alpha} c_{2}(t)
$$

where

$$
E_{\alpha}(z)=\sum_{n=0}^{\infty} \frac{z^{n}}{\Gamma(\alpha n+1)}, \quad E_{\alpha, \mu}(z)=\sum_{n=0}^{\infty} \frac{z^{n}}{\Gamma(\alpha n+\mu)}
$$

and Mittag-Leffler functions. 
By Lemma 2 we can estimate the first term in the right hand side in (2.12).

Let $y(t)=D_{0 t}^{-\alpha}\|u\|_{W_{2}^{1}(0, l)}^{2}, \partial_{0 t}^{\alpha} y(t)=\|u\|_{W_{2}^{1}(0, l)}^{2}$, then

$$
D_{0 t}^{-\alpha}\|u\|_{W_{2}^{1}(0, l)}^{2} \leqslant M_{17}\left(D_{0 t}^{-2 \alpha}\left(\|f\|_{0}^{2}+\mu_{1}^{2}(t)+\mu_{2}^{2}(t)\right)+\left\|u_{0}(x)\right\|_{W_{2}^{1}(0, l)}^{2}\right) .
$$

The following lemma holds.

Lemma 3. Each non-negative integrable on $[0, T]$ function $g(t)$ satisfies the inequality

$$
D_{0 t}^{-2 \alpha} g(t)=\frac{t^{\alpha} \Gamma(\alpha)}{\alpha \Gamma^{2}(\alpha)-\Gamma(2 \alpha)} D_{0 t}^{-\alpha} g(t)
$$

Proof. We transform a fractional integral in the left hand side:

$$
D_{0 t}^{-2 \alpha} g(t)=\frac{1}{\Gamma(2 \alpha)} \int_{0}^{t}(t-\tau)^{2 \alpha-1} g(\tau) d \tau=\frac{1}{\Gamma(2 \alpha)} \int_{0}^{t}(t-\tau)^{\alpha}(t-\tau)^{\alpha-1} g(\tau) d \tau .
$$

Integrating by parts and employing the formula $B(\alpha, \alpha)=\frac{\Gamma^{2}(\alpha)}{\Gamma(2 \alpha)}$, by simple transformations we arrive at (2.14).

Let us show that $\alpha \Gamma^{2}(\alpha)>\Gamma(2 \alpha)$ for all $\alpha \in(0,1)$ in $(2.14)$, or

$$
(2 \alpha) !<2(\alpha !)^{2}, \quad \text { for all } \quad \alpha \in(0,1) \text {. }
$$

In order to do this, we consider the inequality $2^{\alpha^{2}}<2$, which is true for all $\alpha \in(-1,1)$. Then by (2.16) we find

We are going to prove

$$
(2 \alpha) ! \leqslant 2^{\alpha^{2}}(\alpha !)^{2}<2(\alpha !)^{2} \quad \text { for all } \quad \alpha \in(0,1) .
$$

$$
(2 \alpha) ! \leqslant 2^{\alpha^{2}}(\alpha !)^{2} \quad \text { for all } \quad \alpha \in \mathbb{R}
$$

by induction. Indeed, (2.18) is true as $\alpha=0$. Assume that it holds for all $\alpha=n$. Let us prove that (2.18) holds for all $\alpha=n+1$, then we obtain

$$
(2 n+2) ! \leqslant 2^{(n+1)^{2}}((n+1) !)^{2} .
$$

We transform the right hand side of $(2.19)$

$$
(2 n+2) !=2 n !(2 n+1)(2 n+2) \leqslant 2^{n^{2}}(n !)^{2}(2 n+1)(2 n+2) \leqslant 2^{n^{2}} 2^{2 n}(n !)^{2} 2(n+1)^{2} .
$$

This implies:

$$
2 n+1 \leqslant 2^{2 n}(n+1) \quad \text { for all } \quad n \in \mathbb{R} .
$$

The latter identity can be proved by induction.

By means of Lemma 3, (2.12), (2.13), (2.14) we find a needed apriori estimate:

$$
\begin{aligned}
\|u\|_{W_{2}^{1}(0, l)}^{2} & +D_{0 t}^{-\alpha}\left(\left\|u_{x}\right\|_{0}^{2}+\left\|\partial_{0 t}^{\alpha} u\right\|_{0}^{2}+\left\|\partial_{0 t}^{\alpha} u_{x}\right\|_{0}^{2}\right) \\
& \leqslant M\left(D_{0 t}^{-\alpha}\left(\|f\|_{0}^{2}+\mu_{1}^{2}(t)+\mu_{2}^{2}(t)\right)+\left\|u_{0}(x)\right\|_{W_{2}^{1}(0, l)}^{2}\right),
\end{aligned}
$$

where $M$ is a positive constant depending only on data (1.1)-(1.4),

$$
D_{0 t}^{-\alpha} u=\frac{1}{\Gamma(\alpha)} \int_{0}^{t} \frac{u d \tau}{(t-\tau)^{1-\alpha}}
$$

is a fractional Riemann-Liouville integral of order $\alpha, 0<\alpha<1$.

Theorem 1. If $k(x, t) \in C^{1,0}\left(\bar{Q}_{T}\right), \eta(x) \in C^{1}[0, l], r(x, t), q(x, t), f(x, t) \in C\left(\bar{Q}_{T}\right), u(x, t) \in$ $C^{2,0}\left(Q_{T}\right) \cap C^{1,0}\left(\bar{Q}_{T}\right), \partial_{0 t}^{\alpha} u(x, t) \in C\left(\bar{Q}_{T}\right)$ and conditions (1.5) are satisfied, then the solution of problem (1.1)-(1.4) obeys apriori estimate (2.21). 
Apriori estimate (2.21) implies the uniqueness of the solution and the stability in the initial data and the right hand side in the sense of the norm

$$
\|u\|_{1}^{2}=\|u\|_{W_{2}^{1}(0, l)}^{2}+D_{0 t}^{-\alpha}\left(\left\|u_{x}\right\|_{0}^{2}+\left\|\partial_{0 t}^{\alpha} u\right\|_{0}^{2}+\left\|\partial_{0 t}^{\alpha} u_{x}\right\|_{0}^{2}\right)
$$

\section{Stability And COnVERGence of Difference SCHEME}

To solve problem (1.1)-(1.4), we apply the finite element method. We construct a monotone scheme of second order approximation involving the derivatives taking into consideration the sign of $r(x, t)$. In order to do this, instead of equation (1.1), we consider the following equation with perturbed coefficients [28]:

$$
\partial_{0 t}^{\alpha} u=\varkappa\left(k u_{x}\right)_{x}+\partial_{0 t}^{\alpha}\left(\eta u_{x}\right)_{x}+r u_{x}-\int_{0}^{x} q(s, t) u(s, t) d s+f(x, t),
$$

where $\varkappa=\frac{1}{1+R}, R=\frac{0.5 h|r|}{k}$ is the difference Reynolds number.

On the uniform grid $\bar{\omega}_{h \tau}$ we introduce a difference scheme of approximation order $O\left(h^{2}+\tau^{2}\right)$ corresponding to differential problem (1.1)-(1.4):

$$
\begin{aligned}
& \Delta_{0 t_{j+\sigma}}^{\alpha} y=\varkappa_{i}^{j}\left(a_{i}^{j} y_{\bar{x}}^{(\sigma)}\right)_{x}+\Delta_{0 t_{j+\sigma}}^{\alpha}\left(\gamma_{i} y_{\bar{x}}\right)_{x}+b_{i}^{-j} a_{i}^{j} y_{\bar{x}, i}^{(\sigma)}+b_{i}^{+j} a_{i+1}^{j} y_{x, i}^{(\sigma)}-\sum_{s=0}^{i} d_{s}^{j} y_{s}^{(\sigma)} \hbar+\varphi_{i}^{j}, \\
& \varkappa_{0} a_{1} y_{x, 0}^{(\sigma)}+\Delta_{0 t_{j+\sigma}}^{\alpha}\left(\gamma_{1} y_{x, 0}\right)=\beta_{11} y_{0}^{(\sigma)}+0.25 h^{2} d_{0}^{j} y_{0}^{(\sigma)}+\tilde{\beta}_{12} \Delta_{0 t_{j+\sigma}}^{\alpha} y_{0}-\tilde{\mu}_{1}, t \in \bar{\omega}_{\tau}, \\
& -\left(\varkappa_{N} a_{N} y_{\bar{x}, N}^{(\sigma)}+\Delta_{0 t_{j+\sigma}}^{\alpha}\left(\gamma_{N} y_{\bar{x}, N}\right)\right)=\beta_{21} y_{N}^{(\sigma)}+0.5 h \sum_{s=0}^{N} d_{s}^{j} y_{s}^{(\sigma)} \hbar+\tilde{\beta}_{22} \Delta_{0 t_{j+\sigma}}^{\alpha} y_{N}-\tilde{\mu}_{2}, \\
& y(x, 0)=u_{0}(x), \quad x \in \bar{\omega}_{h},
\end{aligned}
$$

where

$$
\begin{aligned}
& \tilde{\beta}_{12}=\beta_{12}+0.5 h, \quad \tilde{\mu}_{1}\left(t_{j+\sigma}\right)=\mu_{1}\left(t_{j+\sigma}\right)+0.5 h \varphi_{0}^{j}, \quad \tilde{\beta}_{22}=\beta_{22}\left(t_{j+\sigma}\right)+0.5 h, \\
& \tilde{\mu}_{2}\left(t_{j+\sigma}\right)=\mu_{2}\left(t_{j+\sigma}\right)+0.5 h \varphi_{N}^{j}, \quad a_{i}^{j}=k\left(x_{i-0.5}, t^{j+\sigma}\right), \quad \gamma_{i}=\eta\left(x_{i-0.5}\right), \\
& b_{i}^{j}=\frac{r\left(x, t_{j+\sigma}\right)}{k\left(x, t_{j+\sigma}\right)}, \quad \varphi=f\left(x_{i}, t_{j+\sigma}\right) \quad y^{(\sigma)}=\sigma y^{j+1}+(1-\sigma) y^{j}, \quad d_{i}^{j}=d\left(x_{i}, t_{j+\sigma}\right), \\
& a_{0}^{(\alpha, \sigma)}=\sigma^{1-\alpha}, \quad a_{l}^{(\alpha, \sigma)}=(l+\sigma)^{1-\alpha}-(l-1+\sigma)^{1-\alpha}, \quad l \geqslant 1, \quad \sigma=1-\frac{\alpha}{2}, \\
& b_{l}^{(\alpha, \sigma)}=\frac{1}{2-\alpha}\left[(l+\sigma)^{2-\alpha}-(l-1+\sigma)^{2-\alpha}\right]-\frac{1}{2}\left[(l+\sigma)^{1-\alpha}+(l-1+\sigma)^{1-\alpha}\right], l \geqslant 1, \\
& c_{0}^{(\alpha, \sigma)}=a_{0}^{(\alpha, \sigma)} \quad \text { as } \quad j=0, \quad \quad \quad l=0, \\
& c_{s}^{(\alpha, \sigma)}= \begin{cases}a_{0}^{(\alpha, \sigma)}+b_{1}^{(\alpha, \sigma)}, & j>0, \\
a_{s}^{(\alpha, \sigma)}+b_{s+1}^{(\alpha, \sigma)}-b_{s}^{(\alpha, \sigma)}, & 1 \leqslant s \leqslant j-1, \\
a_{j}^{(\alpha, \sigma)}-b_{j}^{(\alpha, \sigma)}, & s=j,\end{cases} \\
& c_{s}^{(\alpha, \sigma)}>\frac{1-\alpha}{2}(s+\sigma)^{-\alpha}>0,
\end{aligned}
$$

and

$$
\Delta_{0 t_{j+\sigma}}^{\alpha} y=\frac{\tau^{1-\alpha}}{\Gamma(2-\alpha)} \sum_{s=0}^{j} c_{j-s}^{(\alpha, \sigma)} y_{t}^{s}
$$


is a discrete analogue of Gerasimov-Caputo fractional derivative of order $\alpha, 0<\alpha<1$ [30]. We introduce scalar products and a norm:

$$
[u, v]=\sum_{i=0}^{N} u_{i} v_{i} \hbar, \quad \hbar=\left\{\begin{array}{ll}
0.5 h, & i=0, N, \\
h, & i \neq 0, N,
\end{array} \quad(u, v]=\sum_{i=1}^{N} u_{i} v_{i} h, \quad[u, u]=\left[1, u^{2}\right]=|[u]|_{0}^{2},\right.
$$

We rewrite (3.2)-(3.5) in an operator form:

$$
\begin{aligned}
& \Delta_{0 t_{j+\sigma}}^{\alpha} y=\bar{\Lambda}\left(t_{j+\sigma}\right) y^{(\sigma)}+\bar{\delta} y+\bar{\Phi}, \\
& y(x, 0)=u_{0}(x), \quad x \in \bar{\omega}_{h},
\end{aligned}
$$

where

$$
\begin{aligned}
& \bar{\Lambda}\left(t_{j+\sigma}\right) y^{(\sigma)}=\left\{\begin{array}{l}
\tilde{\Lambda} y_{i}^{(\sigma)}=\varkappa\left(a y_{\bar{x}}^{(\sigma)}\right)_{x}+b^{-} a y_{\bar{x}}^{(\sigma)}+b^{+} a^{(+1)} y_{x}^{(\sigma)}-\sum_{s=0}^{i} d_{s}^{j} y_{s}^{(\sigma)} \hbar, \\
\Lambda^{-} y_{0}^{(\sigma)}=\frac{2}{h}\left(\varkappa_{0} a_{1} y_{x, 0}^{(\sigma)}-\beta_{11} y_{0}^{(\sigma)}-0.25 h^{2} d_{0}^{j} y_{0}^{(\sigma)}\right), \quad i=0, \\
\Lambda^{+} y_{N}^{(\sigma)}=\frac{2}{h}\left(-\varkappa_{N} a_{N} y_{\bar{x}, N}^{(\sigma)}-\beta_{21} y_{N}^{(\sigma)}-0.5 h \sum_{s=0}^{N} d_{s}^{j} y_{s}^{(\sigma)} \hbar\right), \quad i=N,
\end{array}\right. \\
& \bar{\delta} y=\left\{\begin{array}{l}
\delta y_{i}=\Delta_{0 t_{j+\sigma}}^{\alpha}\left(\gamma_{i} y_{\bar{x}}\right)_{x}, \quad i=\overline{1, N-1}, \\
\delta^{-} y_{0}=\frac{2}{h}\left(\Delta_{0 t_{j+\sigma}}^{\alpha}\left(\gamma_{1} y_{x, 0}\right)-\beta_{12} \Delta_{0 t_{j+\sigma}}^{\alpha} y_{0}\right), \quad i=0, \\
\delta^{+} y_{N}=\frac{2}{h}\left(-\Delta_{0 t_{j+\sigma}}^{\alpha}\left(\gamma_{N} y_{\bar{x}, N}\right)-\beta_{22} \Delta_{0 t_{j+\sigma}}^{\alpha} y_{N}\right), \quad i=N
\end{array}\right.
\end{aligned}
$$

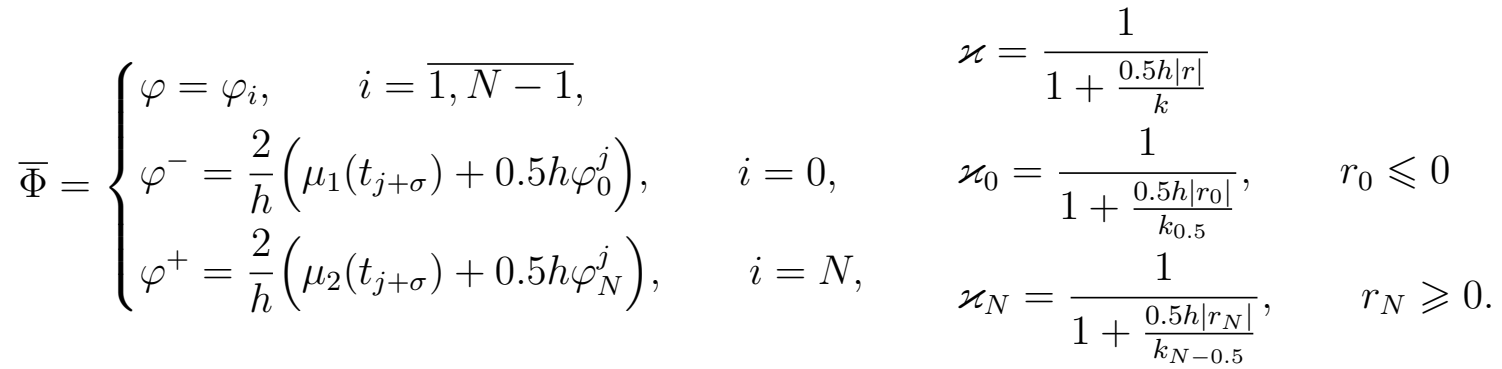

We calculate the scalar product of (3.6) with $\bar{y}=y^{(\sigma)}+\Delta_{0 t_{j+\sigma}}^{\alpha} y$ :

$$
\left[\Delta_{0 t_{j+\sigma}}^{\alpha} y, \bar{y}\right]=\left[\bar{\Lambda}\left(t_{j+\sigma}\right) y^{(\sigma)}, \bar{y}\right]+[\bar{\delta} y, \bar{y}]+[\bar{\Phi}, \bar{y}]
$$

The following lemma holds [30].

Lemma 4. Each function $y(t)$ defined on the grid $\bar{\omega}_{\tau}$ satisfies the inequality:

$$
y^{(\sigma)} \Delta_{0 t_{j+\sigma}}^{\alpha} y \geqslant \frac{1}{2} \Delta_{0 t_{j+\sigma}}^{\alpha}\left(y^{2}\right)
$$

By means of Lemma 4, we estimate the sums in (3.8):

$$
\begin{aligned}
{\left[\Delta_{0 t_{j+\sigma}}^{\alpha} y, \bar{y}\right] } & =\left[\Delta_{0 t_{j+\sigma}}^{\alpha} y, y^{(\sigma)}+\Delta_{0 t_{j+\sigma}}^{\alpha} y\right]=\left[\Delta_{0 t_{j+\sigma}}^{\alpha} y, y^{(\sigma)}\right]+\left[1,\left(\Delta_{0 t_{j+\sigma}}^{\alpha} y\right)^{2}\right] \\
& \geqslant \frac{1}{2} \Delta_{0 t_{j+\sigma}}^{\alpha}|[y]|_{0}^{2}+\left|\left[\Delta_{0 t_{j+\sigma}}^{\alpha} y\right]\right|_{0}^{2}
\end{aligned}
$$




$$
\begin{aligned}
{\left[\bar{\Lambda}\left(t_{j+\sigma}\right) y^{(\sigma)}, \bar{y}\right]=} & \left(\tilde{\Lambda} y^{(\sigma)}, \bar{y}\right)+0.5 h \bar{y}_{0} \Lambda^{-} y_{0}^{(\sigma)}+0.5 h \bar{y}_{N} \Lambda^{+} y_{N}^{(\sigma)} \\
= & \left(\varkappa\left(a y_{\bar{x}}^{(\sigma)}\right)_{x}, \bar{y}\right)+\left(b^{-} a y_{\bar{x}}^{(\sigma)}, \bar{y}\right)+\left(b^{+} a^{(+1)} y_{x}^{(\sigma)}, \bar{y}\right) \\
& -\left(\sum_{s=0}^{i} d_{s}^{j} y_{s}^{(\sigma)} \hbar, \bar{y}\right)+\bar{y}_{0} \varkappa_{0} a_{1} y_{x, 0}^{(\sigma)}-\beta_{11} y_{0}^{(\sigma)} \bar{y}_{0} \\
& -\varkappa_{N} a_{N} y_{\bar{x}, N}^{(\sigma)} \bar{y}_{N}-0.25 h^{2} d_{0}^{j} y_{0}^{(\sigma)} \bar{y}_{0} \\
& -\beta_{21} y_{N}^{(\sigma)} \bar{y}_{N}-0.5 h d_{N} \bar{y}_{N} \sum_{s=0}^{N} d_{s}^{j} y_{s}^{(\sigma)} \hbar .
\end{aligned}
$$

We transform the terms in the right hand side in (3.10):

$$
\begin{aligned}
& \left(\varkappa\left(a y_{\bar{x}}^{(\sigma)}\right)_{x}, \bar{y}\right)=\left.\bar{y} \varkappa a y_{\bar{x}}^{(\sigma)}\right|_{0} ^{N}-\left(a y_{\bar{x}}^{(\sigma)},(\varkappa \bar{y})_{\bar{x}}\right] \\
& =\bar{y}_{N} \varkappa_{N} a_{N} y_{\bar{x}, N}^{(\sigma)}-\bar{y}_{0} \varkappa_{0} a_{1} y_{x, 0}^{(\sigma)}-\left(a y_{\bar{x}}^{(\sigma)}, \varkappa_{\bar{x}} \bar{y}+\varkappa^{(-1)} \bar{y}_{\bar{x}}\right] \\
& =\bar{y}_{N} \varkappa_{N} a_{N} y_{\bar{x}, N}^{(\sigma)}-\bar{y}_{0} \varkappa_{0} a_{1} y_{x, 0}^{(\sigma)}-\left(a y_{\bar{x}}^{(\sigma)}, \varkappa_{\bar{x}} y^{(\sigma)}\right]-\left(a y_{\bar{x}}^{(\sigma)}, \varkappa_{\bar{x}} \Delta_{0 t_{j+\sigma}}^{\alpha} y\right] \\
& -\left(a y_{\bar{x}}^{(\sigma)}, \varkappa^{(-1)} y_{\bar{x}}^{(\sigma)}\right]-\left(a y_{\bar{x}}^{(\sigma)}, \varkappa^{(-1)} \Delta_{0 t_{j+\sigma}}^{\alpha} y_{\bar{x}}\right] \\
& \leqslant \bar{y}_{N} \varkappa_{N} a_{N} y_{\bar{x}, N}^{(\sigma)}-\bar{y}_{0} \varkappa_{0} a_{1} y_{x, 0}^{(\sigma)}+\varepsilon\left|\left[\Delta_{0 t_{j+\sigma}}^{\alpha} y\right]\right|_{0}^{2}+M_{1}^{\varepsilon}\left(\left|\left[y^{(\sigma)}\right]\right|_{0}^{2}+\left|\left[y_{\bar{x}}^{(\sigma)}\right]\right|_{0}^{2}\right) \\
& -\frac{1}{1+h M_{2}}\left(a \varkappa,\left(y_{\bar{x}}^{(\sigma)}\right)^{2}\right]-\frac{c_{0}}{2\left(1+h M_{2}\right)}\left(\varkappa, \Delta_{0 t_{j+\sigma}}^{\alpha} y_{\bar{x}}^{2}\right] \\
& \left.\leqslant \bar{y}_{N} \varkappa_{N} a_{N} y_{\bar{x}, N}^{(\sigma)}-\bar{y}_{0} \varkappa_{0} a_{1} y_{x, 0}^{(\sigma)}+\varepsilon\left|\left[\Delta_{0 t_{j+\sigma}}^{\alpha} y\right]\right|_{0}^{2}+\left.M_{1}^{\varepsilon}\left(\left|\left[y^{(\sigma)}\right]\right|_{0}^{2}+\| y_{\bar{x}}^{(\sigma)}\right]\right|_{0} ^{2}\right) \\
& \left.\left.-M_{3} \| y_{\bar{x}}^{(\sigma)}\right]\left.\right|_{0} ^{2}-M_{4} \Delta_{0 t_{j+\sigma}}^{\alpha} \| y_{\bar{x}}\right]\left.\right|_{0} ^{2}, \\
& \left(b^{-} a y_{\bar{x}}^{(\sigma)}, \bar{y}\right)+\left(b^{+} a^{(+1)} y_{x}^{(\sigma)}, \bar{y}\right)=\left(b^{-} a y_{\bar{x}}^{(\sigma)}, y^{(\sigma)}\right)+\left(b^{-} a y_{\bar{x}}^{(\sigma)}, \Delta_{0 t_{j+\sigma}}^{\alpha} y\right) \\
& +\left(b^{+} a^{(+1)} y_{x}^{(\sigma)}, y^{(\sigma)}\right)+\left(b^{+} a^{(+1)} y_{x}^{(\sigma)}, \Delta_{0 t_{j+\sigma}}^{\alpha} y\right) \\
& \left.\leqslant \varepsilon\left|\left[\Delta_{0 t_{j+\sigma}}^{\alpha} y\right]\right|_{0}^{2}+\left.M_{5}^{\varepsilon}\left(\left|\left[y^{(\sigma)}\right]\right|_{0}^{2}+\| y_{\bar{x}}^{(\sigma)}\right]\right|_{0} ^{2}\right) \text {, } \\
& -\left(\sum_{s=0}^{i} d_{s}^{j} y_{s}^{(\sigma)} \hbar, \bar{y}\right)-0.25 h^{2} d_{0}^{j} y_{0}^{(\sigma)} \bar{y}_{0}-0.5 h d_{N} \bar{y}_{N} \sum_{s=0}^{N} d_{s}^{j} y_{s}^{(\sigma)} \hbar-\beta_{11} y_{0}^{(\sigma)} \bar{y}_{0}-\beta_{21} y_{N}^{(\sigma)} \bar{y}_{N} \\
& =-\left[\sum_{s=0}^{i} d_{s}^{j} y_{s}^{(\sigma)} \hbar, y^{(\sigma)}\right]-\left[\sum_{s=0}^{i} d_{s}^{j} y_{s}^{(\sigma)} \hbar, \Delta_{0 t_{j+\sigma}}^{\alpha} y\right] \\
& -\beta_{11}\left(y_{0}^{(\sigma)}\right)^{2}-\beta_{11} y_{0}^{(\sigma)} \Delta_{0 t_{j+\sigma}}^{\alpha} y_{0} \\
& -\beta_{21}\left(y_{N}^{(\sigma)}\right)^{2}-\beta_{21} y_{0}^{(\sigma)} \Delta_{0 t_{j+\sigma}}^{\alpha} y_{N} \\
& \leqslant \varepsilon_{1}\left|\left[\Delta_{0 t_{j+\sigma}}^{\alpha} y\right]\right|_{0}^{2}+M_{6}^{\varepsilon_{1}}\left[1,\left(\sum_{s=0}^{i} d_{s}^{j} y_{s}^{(\sigma)} \hbar\right)^{2}\right]+M_{7}^{\varepsilon_{2}, \varepsilon_{3}}\left|\left[y^{(\sigma)}\right]\right|_{0}^{2} \\
& +\varepsilon_{2}\left(\Delta_{0 t_{j+\sigma}}^{\alpha} y_{0}\right)^{2}+\varepsilon_{3}\left(\Delta_{0 t_{j+\sigma}}^{\alpha} y_{N}\right)^{2} \\
& \leqslant \varepsilon_{1}\left|\left[\Delta_{0 t_{j+\sigma}}^{\alpha} y\right]\right|_{0}^{2}+M_{8}^{\varepsilon_{1}, \varepsilon_{2}, \varepsilon_{3}}\left|\left[y^{(\sigma)}\right]\right|_{0}^{2}+\varepsilon_{2}\left(\Delta_{0 t_{j+\sigma}}^{\alpha} y_{0}\right)^{2}+\varepsilon_{3}\left(\Delta_{0 t_{j+\sigma}}^{\alpha} y_{N}\right)^{2} .
\end{aligned}
$$


In view of (3.11)-(3.13), by (3.10) we obtain

$$
\begin{aligned}
& {\left[\bar{\Lambda}\left(t_{j+\sigma}\right) y^{(\sigma)}, \bar{y}\right] \leqslant \varepsilon_{1}\left|\left[\Delta_{0 t_{j+\sigma}}^{\alpha} y\right]\right|_{0}^{2}+\varepsilon_{2}\left(\Delta_{0 t_{j+\sigma}}^{\alpha} y_{0}\right)^{2}+\varepsilon_{3}\left(\Delta_{0 t_{j+\sigma}}^{\alpha} y_{N}\right)^{2}} \\
& \left.\left.\left.+\left.M_{7}^{\varepsilon_{1}, \varepsilon_{2}, \varepsilon_{3}}\left(\left|\left[y^{(\sigma)}\right]\right|_{0}^{2}+\| y_{\bar{x}}^{(\sigma)}\right]\right|_{0} ^{2}\right)-M_{3} \| y_{\bar{x}}^{(\sigma)}\right]\left.\right|_{0} ^{2}-M_{4} \Delta_{0 t_{j+\sigma}}^{\alpha} \| y_{\bar{x}}\right]\left.\right|_{0} ^{2}, \\
& {[\bar{\delta} y, \bar{y}]=(\delta y, \bar{y})+0.5 h \delta^{-} y_{0} \bar{y}_{0}+0.5 h \delta^{+} y_{N} \bar{y}_{N}} \\
& =\left(\Delta_{0 t_{j+\sigma}}^{\alpha}\left(\gamma_{i} y_{\bar{x}}\right)_{x}, \bar{y}\right)+\bar{y}_{0} \Delta_{0 t_{j+\sigma}}^{\alpha}\left(\gamma_{i} y_{x, 0}\right)-\bar{y}_{0} \beta_{12} \Delta_{0 t_{j+\sigma}}^{\alpha} y_{0} \\
& -\bar{y}_{N} \Delta_{0 t_{j+\sigma}}^{\alpha}\left(\gamma_{N} y_{\bar{x}, N}\right)-\bar{y}_{N} \beta_{22} \Delta_{0 t_{j+\sigma}}^{\alpha} y_{N} \\
& =-\left(\Delta_{0 t_{j+\sigma}}^{\alpha}\left(\gamma_{i} y_{\bar{x}}\right), \bar{y}_{\bar{x}}\right]-\beta_{12} \bar{y}_{0} \Delta_{0 t_{j+\sigma}}^{\alpha} y_{0}-\bar{y}_{N} \beta_{22} \Delta_{0 t_{j+\sigma}}^{\alpha} y_{N} \\
& =-\left(\Delta_{0 t_{j+\sigma}}^{\alpha}\left(\gamma_{i} y_{\bar{x}}^{(\sigma)}\right), y_{\bar{x}}^{(\sigma)}\right]-\left(\gamma_{i},\left(\Delta_{0 t_{j+\sigma}}^{\alpha} y_{\bar{x}}\right)^{2}\right]-\beta_{12} y_{0}^{(\sigma)} \Delta_{0 t_{j+\sigma}}^{\alpha} y_{0} \\
& -\beta_{12}\left(\Delta_{0 t_{j+\sigma}}^{\alpha} y_{0}\right)^{2}--\beta_{22} y_{N}^{(\sigma)} \Delta_{0 t_{j+\sigma}}^{\alpha} y_{N}-\beta_{22}\left(\Delta_{0 t_{j+\sigma}}^{\alpha} y_{N}\right)^{2} \\
& \left.\left.\leqslant-\frac{c_{0}}{2} \Delta_{0 t_{j+\sigma}}^{\alpha} \| y_{\bar{x}}\right]\left.\right|_{0} ^{2}-c_{0} \| \Delta_{0 t_{j+\sigma}}^{\alpha} y_{\bar{x}}\right]\left.\right|_{0} ^{2}-\frac{\beta_{12}}{2} \Delta_{0 t_{j+\sigma}}^{\alpha} y_{0}^{2} \\
& -\frac{\beta_{22}}{2} \Delta_{0 t_{j+\sigma}}^{\alpha} y_{N}^{2}-\beta_{12}\left(\Delta_{0 t_{j+\sigma}}^{\alpha} y_{0}\right)^{2}-\beta_{22}\left(\Delta_{0 t_{j+\sigma}}^{\alpha} y_{N}\right)^{2} \\
& {[\bar{\Phi}, \bar{y}]=(\varphi, \bar{y})+0.5 h \varphi^{-} \bar{y}_{0}+0.5 h \varphi^{+} \bar{y}_{N}} \\
& =(\varphi, \bar{y})+\bar{y}_{0}\left(\mu_{1}+0.5 h \varphi_{0}\right)+\bar{y}_{N}\left(\mu_{2}+0.5 h \varphi_{N}\right) \\
& =(\varphi, \bar{y})+\bar{y}_{0} \mu_{1}+0.5 h \varphi_{0} \bar{y}_{0}+\bar{y}_{N} \mu_{2}+0.5 h \varphi_{N} \bar{y}_{N} \\
& =[\varphi, \bar{y}]+\mu_{1} \bar{y}_{0}+\mu_{2} \bar{y}_{N} \\
& =\left[\varphi, y^{(\sigma)}\right]+\left[\varphi, \Delta_{0 t_{j+\sigma}}^{\alpha} y\right]+\mu_{1} y_{0}^{(\sigma)}+\mu_{1} \Delta_{0 t_{j+\sigma}}^{\alpha} y_{0}+\mu_{2} y_{N}^{(\sigma)}+\mu_{2} \Delta_{0 t_{j+\sigma}}^{\alpha} y_{N} \\
& \leqslant \varepsilon_{1}\left|\left[\Delta_{0 t_{j+\sigma}}^{\alpha} y\right]\right|_{0}^{2}+\varepsilon_{2}\left(\Delta_{0 t_{j+\sigma}}^{\alpha} y_{0}\right)^{2}+\varepsilon_{3}\left(\Delta_{0 t_{j+\sigma}}^{\alpha} y_{N}\right)^{2}+M_{9}^{\varepsilon_{2}, \varepsilon_{3}}\left(\mu_{1}^{2}+\mu_{2}^{2}\right) \\
& \left.+\left.M_{10}\left(\left|\left[y^{(\sigma)}\right]\right|_{0}^{2}+\| y_{\bar{x}}^{(\sigma)}\right]\right|_{0} ^{2}\right)+M_{11}^{\varepsilon_{1}}|[\varphi]|_{0}^{2} .
\end{aligned}
$$

Due to (3.9)-(3.16), by (3.8) we find:

$$
\begin{aligned}
\frac{1}{2} \Delta_{0 t_{j+\sigma}}^{\alpha}|[y]|_{0}^{2} & \left.\left.\left.+\left|\left[\Delta_{0 t_{j+\sigma}}^{\alpha} y\right]\right|_{0}^{2}+M_{12} \Delta_{0 t_{j+\sigma}}^{\alpha} \| y_{\bar{x}}\right]\left.\right|_{0} ^{2}+c_{0} \| \Delta_{0 t_{j+\sigma}}^{\alpha} y_{\bar{x}}\right]\left.\right|_{0} ^{2}+M_{3} \| y_{\bar{x}}^{(\sigma)}\right]\left.\right|_{0} ^{2} \\
& +\frac{\beta_{12}}{2} \Delta_{0 t_{j+\sigma}}^{\alpha} y_{0}^{2}+\frac{\beta_{22}}{2} \Delta_{0 t_{j+\sigma}}^{\alpha} y_{N}^{2}+\beta_{12}\left(\Delta_{0 t_{j+\sigma}}^{\alpha} y_{0}\right)^{2}+\beta_{22}\left(\Delta_{0 t_{j+\sigma}}^{\alpha} y_{N}\right)^{2} \\
\leqslant & \varepsilon_{1}\left|\left[\Delta_{0 t_{j+\sigma}}^{\alpha} y\right]\right|_{0}^{2}+\varepsilon_{2}\left(\Delta_{0 t_{j+\sigma}}^{\alpha} y_{0}\right)^{2}+\varepsilon_{3}\left(\Delta_{0 t_{j+\sigma}}^{\alpha} y_{N}\right)^{2} \\
& \left.+\left.M_{13}^{\varepsilon_{1}, \varepsilon_{2}, \varepsilon_{3}}\left(\left|\left[y^{(\sigma)}\right]\right|_{0}^{2}+\| y_{\bar{x}}^{(\sigma)}\right]\right|_{0} ^{2}\right)+M_{14}^{\varepsilon_{2}, \varepsilon_{3}}\left(\mu_{1}^{2}+\mu_{2}^{2}\right)+M_{11}^{\varepsilon_{1}} \mid[\varphi \mid]_{0}^{2}
\end{aligned}
$$

Choosing $\varepsilon_{1}=\frac{1}{2}, \varepsilon_{2}=\frac{\beta_{12}}{2}, \varepsilon_{3}=\frac{\beta_{22}}{2}$, by (3.17) we get:

$$
\begin{aligned}
\Delta_{0 t_{j+\sigma}}^{\alpha}|[y]|_{W_{2}^{1}(0, l)}^{2} & \left.\left.+\| y_{\bar{x}}^{(\sigma)}\right]\left.\right|_{0} ^{2}+\left|\left[\Delta_{0 t_{j+\sigma}}^{\alpha} y\right]\right|_{0}^{2}+\| \Delta_{0 t_{j+\sigma}}^{\alpha} y_{\bar{x}}\right]\left.\right|_{0} ^{2} \\
& \leqslant M_{15}\left|\left[y^{(\sigma)}\right]\right|_{W_{2}^{1}(0, l)}^{2}+M_{16}\left(|[\varphi]|_{0}^{2}+\mu_{1}^{2}+\mu_{2}^{2}\right)
\end{aligned}
$$


where $\left.|[y]|_{W_{2}^{1}(0, l)}^{2}=|[y]|_{0}^{2}+\| y_{\bar{x}}\right]\left.\right|_{0} ^{2}$. We rewrite $(3.18)$ as

$$
\Delta_{0 t_{j+\sigma}}^{\alpha}|[y]|_{W_{2}^{1}(0, l)}^{2} \leqslant M_{17}^{\sigma}\left|\left[y^{j+1}\right]\right|_{W_{2}^{1}(0, l)}^{2}+M_{18}^{\sigma}\left|\left[y^{j}\right]\right|_{W_{2}^{1}(0, l)}^{2}+M_{16}\left(|[\varphi]|_{0}^{2}+\mu_{1}^{2}+\mu_{2}^{2}\right) .
$$

The following lemma holds true.

Lemma 5. Let $\left\{p_{j}\right\}$ be a sequence obeying the following conditions:

$$
p_{0}=1, \quad \bar{\sigma}^{1-\alpha} p_{j}=\sum_{s=1}^{j}\left(c_{s-1}^{\alpha, \sigma}-c_{s}^{\alpha, \sigma}\right) p_{j-s}, \quad j \geqslant 1,
$$

then

$$
0<p_{j}<1, \quad \sum_{s=k}^{j} p_{j-s} c_{s-k}^{\alpha, \sigma}=\bar{\sigma}^{1-\alpha}, \quad 1 \leqslant k \leqslant j
$$

where

$$
\bar{\sigma}^{1-\alpha}=\frac{1}{2-\alpha}\left((1+\sigma)^{2-\alpha}-\sigma^{2-\alpha}\right)-\frac{1}{2}\left((1+\sigma)^{1-\alpha}-\sigma^{1-\alpha}\right) .
$$

Proof. We follow [31] to prove identity (3.20). Since $c_{s}<c_{s-1}$ as $s \geqslant 1$, we obtain:

$$
\sum_{s=1}^{j} p_{j-s} c_{s}^{\alpha, \sigma}<\sum_{s=1}^{j} p_{j-s} c_{s-1}^{\alpha, \sigma}
$$

where

$$
\sum_{s=1}^{j} p_{j-s} c_{s-1}^{\alpha, \sigma}=\sum_{s=0}^{j} p_{j-s} c_{s}^{\alpha, \sigma}
$$

By (3.21), (3.22) we find

$$
\sum_{s=j}^{j} p_{j-s} c_{s-j}^{\alpha, \sigma}=p_{0} c_{0}=\bar{\sigma}^{1-\alpha}
$$

where

$$
\begin{aligned}
& \bar{\sigma}^{1-\alpha}= \begin{cases}\sigma^{1-\alpha}, & j=0 \\
\frac{1}{2-\alpha}\left((1+\sigma)^{2-\alpha}-\sigma^{2-\alpha}\right)-\frac{1}{2}\left((1+\sigma)^{1-\alpha}-\sigma^{1-\alpha}\right), & j \geqslant 1,\end{cases} \\
& \sum_{s=1}^{j} p_{j-s} c_{s}^{\alpha, \sigma}<\sum_{s=0}^{j} p_{j-s} c_{s}^{\alpha, \sigma}=p_{0} c_{0}=\bar{\sigma}^{1-\alpha} .
\end{aligned}
$$

Thanks to (3.23), (3.24), we obtain

$$
\begin{aligned}
& \sum_{s=1}^{j} p_{j-s} c_{s-1}^{\alpha, \sigma}=\bar{\sigma}^{1-\alpha}, \quad \sum_{s=1}^{j} p_{j-s} c_{s}^{\alpha, \sigma}<\bar{\sigma}^{1-\alpha}, \quad \sum_{s=1}^{j} p_{j-s}\left(c_{s-1}^{\alpha, \sigma}-c_{s}^{\alpha, \sigma}\right)<\bar{\sigma}^{1-\alpha}, \\
& \sum_{s=1}^{j} p_{j-s} c_{s}^{\alpha, \sigma}<\sum_{s=1}^{j} p_{j-s} c_{s}^{\alpha, \sigma}+p_{j} c_{0}, \quad p_{j} c_{0}>0, \quad c_{0}=\bar{\sigma}^{1-\alpha} .
\end{aligned}
$$

By (3.22) we find

$$
c_{0} p_{j}=\sum_{s=1}^{j}\left(c_{s-1}^{\alpha, \sigma}-c_{s}^{\alpha, \sigma}\right) p_{j-s} .
$$

It follows from (3.24), (3.25) that

$$
0<p_{j} c_{0}<\bar{\sigma}^{1-\alpha}, \quad 0<p_{j}<1 .
$$


Let $s=l+k-1$, then by (3.23) we obtain:

$$
\sum_{s=k}^{j} p_{j-s} c_{s-k}^{\alpha, \sigma}=\sum_{l=1}^{j-k+1} p_{j-k+1-l} c_{l-1}^{\alpha, \sigma}=\bar{\sigma}^{1-\alpha}, \quad 1 \leqslant k \leqslant j .
$$

The proof is complete.

Lemma 6. Assume that (3.20) holds, then

$$
\frac{\Gamma(2-\alpha)}{\Gamma(1+(m-1) \alpha)} \sum_{s=1}^{j} p_{j-s} s^{(m-1) \alpha} \leqslant \frac{\bar{\sigma}^{1-\alpha} j^{m \alpha}}{\Gamma(1+m \alpha)}, \quad m \in \mathbb{N} .
$$

The proof of this lemma is similar to that of Lemma 3.2 in 31 .

Lemma 7. Let $\vec{e}=(1,1, \ldots, 1)^{T} \in R^{j}$,

$$
J=2 \bar{\sigma}^{\alpha-1} \Gamma(2-\alpha) \lambda \tau^{\alpha}\left[\begin{array}{ccccc}
0 & p_{1} & \ldots & p_{j-2} & p_{j-1} \\
0 & 0 & \ldots & p_{j-3} & p_{j-2} \\
& & \ldots & & \\
0 & 0 & \ldots & 0 & p_{1} \\
0 & 0 & \ldots & 0 & 0
\end{array}\right]_{j \times j}
$$

and (3.26) holds. Then

$$
\begin{aligned}
& J^{i}=0, \quad i \geqslant j, \\
& J^{m} \vec{e} \leqslant \frac{1}{\Gamma(1+m \alpha)}\left(\left(2 \lambda t_{j}^{\alpha}\right)^{m},\left(2 \lambda t_{j-1}^{\alpha}\right)^{m}, \ldots,\left(2 \lambda t_{1}^{\alpha}\right)^{m}\right)^{T}, \quad m=0,1,2, \ldots \\
& \sum_{s=0}^{i} J^{s} \vec{e}=\sum_{s=0}^{j-1} J^{s} \vec{e} \leqslant\left(E_{\alpha}\left(2 \lambda t_{j}^{\alpha}\right), E_{\alpha}\left(2 \lambda t_{j-1}^{\alpha}\right), \ldots, E_{\alpha}\left(2 \lambda t_{1}^{\alpha}\right)\right)^{T}, \quad i \geqslant j .
\end{aligned}
$$

The proof of the above lemma is similar to that of Lemma 3.3 in [31].

Lemma 8. Assume that non-negative sequences $y^{j}, \varphi^{j}, j=0,1,2, \ldots$, satisfy the inequality

$$
\Delta_{0 t_{j+\sigma}}^{\alpha} y^{j} \leqslant \lambda_{1} y^{j+1}+\lambda_{2} y^{j}+\varphi^{j}, \quad j \geqslant 1
$$

where $\lambda_{1} \geqslant 0, \lambda_{2} \geqslant 0$ are constants. Then there exist $\tau_{0}$ such that as $\tau \leqslant \tau_{0}$, we have

$$
y^{j+1} \leqslant 2\left(y^{0}+\frac{t_{j}^{\alpha}}{\Gamma(1+\alpha)} \max _{0 \leqslant j^{\prime} \leqslant j} \varphi^{j^{\prime}}\right) E_{\alpha}\left(2 \lambda t_{j}^{\alpha}\right), \quad 1 \leqslant j \leqslant j_{0},
$$

where

$$
E_{\alpha}(z)=\sum_{k=0}^{\infty} \frac{z^{k}}{\Gamma(1+k \alpha)}
$$

is a Mittag-Leffler function and $\lambda=\lambda_{1}+\frac{\lambda_{2}}{2+2^{1-\alpha}}$.

This lemma can be proved by employing Lemmata 4-6 similar to Lemma 3.1 in [31].

By Lemma 8 and (3.18) we obtain

$$
\left|\left[y^{j+1}\right]\right|_{W_{2}^{1}(0, l)}^{2} \leqslant M\left(\left|\left[y^{0}\right]\right|_{W_{2}^{1}(0, l)}^{2}+\frac{t_{j}^{\alpha}}{\Gamma(1+\alpha)} \max _{0 \leqslant j^{\prime} \leqslant j}\left(\left|\left[\varphi^{j^{\prime}}\right]\right|_{0}^{2}+\mu_{1}^{2}+\mu_{2}^{2}\right)\right),
$$

where $M$ is a positive constant independent of $h$ and $\tau$.

Theorem 2. Assume that conditions (1.5) are satisfied, then there exists $\tau_{0}$ such that as $\tau \leqslant \tau_{0}$, the solution of difference problem (3.2)-(3.5) obeys apriori estimate (3.27). 
Apriori estimate (3.27) implies the uniqueness of solution to problem (3.2)-(3.5) and its stability in initial data and the right hand side.

Let $u(x, t)$ be the solution of problem (1.1)-(1.4), $y\left(x_{i}, t_{j}\right)=y_{i}^{j}$ be the solution of difference problem (3.2)-(3.5). To estimate the exactness of difference scheme (3.2)-(3.5), we consider the difference $z_{i}^{j}=y_{i}^{j}-u_{i}^{j}$, where $u_{i}^{j}=u\left(x_{i}, t_{j}\right)$. Substituting $y=z+u$ into relations (3.2)-(3.5), we obtain a problem for the function $z$ :

$$
\begin{aligned}
& \Delta_{0 t_{j+\sigma}}^{\alpha} z=\varkappa_{i}^{j}\left(a_{i}^{j} z_{\bar{x}}^{(\sigma)}\right)_{x}+\Delta_{0 t_{j+\sigma}}^{\alpha}\left(\gamma_{i} z_{\bar{x}}\right)_{x}+b_{i}^{-j} a_{i}^{j} z_{\bar{x}, i}^{(\sigma)}+b_{i}^{+j} a_{i+1}^{j} z_{x, i}^{(\sigma)}-\sum_{s=0}^{i} d_{s}^{j} z_{s}^{(\sigma)} \hbar+\Psi_{i}^{j}, \\
& \varkappa_{0} a_{1} z_{x, 0}^{(\sigma)}+\Delta_{0 t_{j+\sigma}}^{\alpha}\left(\gamma_{1} z_{x, 0}\right)=\beta_{11} z_{0}^{(\sigma)}+0.25 h^{2} d_{0}^{j} z_{0}^{(\sigma)}+\tilde{\beta}_{12} \Delta_{0 t_{j+\sigma}}^{\alpha} z_{0}-\tilde{\nu}_{1}, t \in \bar{\omega}_{\tau}, \\
& -\left(\varkappa_{N} a_{N} z_{\bar{x}, N}^{(\sigma)}+\Delta_{0 t_{j+\sigma}}^{\alpha}\left(\gamma_{N} z_{\bar{x}, N}\right)\right)=\beta_{21} z_{N}^{(\sigma)}+0.5 h \sum_{s=0}^{N} d_{s}^{j} z_{s}^{(\sigma)} \hbar+\tilde{\beta}_{22} \Delta_{0 t_{j+\sigma}}^{\alpha} z_{N}-\tilde{\nu}_{2}, \\
& z(x, 0)=0, \quad x \in \bar{\omega}_{h},
\end{aligned}
$$

where $\Psi=O\left(h^{2}+\tau^{2}\right), \tilde{\nu}_{1}=O\left(h^{2}+\tau^{2}\right), \tilde{\nu}_{2}=O\left(h^{2}+\tau^{2}\right)$ are the errors of approximation of differential problem (1.1)-(1.4) by difference scheme (3.2)-(3.5) in the class of solutions $u=u(x, t)$ of problem $(1.1)-(1.4)$.

Applying apriori estimate (3.27) to the solution of problem (3.28)-(3.31), we obtain the inequality

$$
\left|\left[z^{j+1}\right]\right|_{W_{2}^{1}(0, l)}^{2} \leqslant M \max _{0 \leqslant j^{\prime} \leqslant j}\left(\left|\left[\Psi^{j^{\prime}}\right]\right|_{0}^{2}+\nu_{1}^{j^{\prime} 2}+\nu_{2}^{j^{\prime} 2}\right)
$$

where $M$ is a positive constant independent of $h$ and $\tau$.

Apriori estimate (3.32) implies the convergence of the solution of difference problem (3.2)(3.5) to the solution of differential problem (1.1)-(1.4) in the sense of the norm $\left|\left[z^{j+1}\right]\right|_{W_{2}^{1}(0, l)}^{2}$ on each fiver so that there exists $\tau_{0}$ such that as $\tau \leqslant \tau_{0}$, the estimate holds:

$$
\left|\left[y^{j+1}-u^{j+1}\right]\right|_{W_{2}^{1}(0, l)}^{2} \leqslant M\left(h^{2}+\tau^{2}\right) .
$$

Corollary 1. The results obtained in the present work holds also for the case, when equation (1.1) reads as

$$
\begin{aligned}
\partial_{0 t}^{\alpha} u= & \frac{\partial}{\partial x}\left(k(x, t) \frac{\partial u}{\partial x}\right)+\partial_{0 t}^{\alpha} \frac{\partial}{\partial x}\left(\eta(x) \frac{\partial u}{\partial x}\right)+r(x, t) \frac{\partial u}{\partial x} \\
& -\int_{0}^{l} q(x, t) u(x, t) d x+f(x, t), \quad 0<x<l, \quad 0<t \leqslant T,
\end{aligned}
$$

if we assume that $|q| \leqslant c_{2}$.

\section{Boundary VAlue PROBlem FOR A DEGENERATING PSEUdO-PARABOLIC EQUATION} WITH A NONLOCAL LINEAR SOURCE

In a closed cylinder $\bar{Q}_{T}=\{(x, t): 0 \leqslant x \leqslant l, 0 \leqslant t \leqslant T\}$ we consider the following nonlocal boundary value problem:

$$
\begin{aligned}
\partial_{0 t}^{\alpha} u= & \frac{1}{x^{m}} \frac{\partial}{\partial x}\left(x^{m} k \frac{\partial u}{\partial x}\right)+\frac{1}{x^{m}} \partial_{0 t}^{\alpha} \frac{\partial}{\partial x}\left(x^{m} \eta(x) \frac{\partial u}{\partial x}\right) \\
& +r \frac{\partial u}{\partial x}-\int_{0}^{x} q(s, t) u(s, t) d s+f(x, t), \quad 0<x<l, \quad 0<t \leqslant T, \\
\lim _{x \rightarrow 0} x^{m} \Pi(x, t)=0, \quad 0 \leqslant t \leqslant T, & \\
-\Pi(l, t) & =\beta_{1}(t) u(l, t)+\beta_{2}(t) \partial_{0 t}^{\alpha} u(l, t)-\mu(t), \quad 0 \leqslant t \leqslant T, \\
u(x, 0) & =u_{0}(x), \quad 0 \leqslant x \leqslant l,
\end{aligned}
$$


where $0 \leqslant m \leqslant 2$. At $x=0$, we assume the boundedness of the solution $|u(0, t)|<\infty$ that is equivalent to condition (4.2), which is equivalent to the identity $\Pi(0, t)=0$ [25] if the functions $r(0, t), k(0, t), q(0, t), f(0, t)$ are finite.

\section{APRIORI ESTIMATE IN DIFFERENTIAL FORM}

We are going to obtain an apriori estimate by the method of energy inequalities. In order to do this, we calculate the scalar product of equation (4.1) with $x^{m} U=x^{m}\left(u+\partial_{0 t}^{\alpha} u\right)$ :

$$
\begin{aligned}
\left(\partial_{0 t}^{\alpha} u, x^{m} U\right)= & \left(\left(x^{m} k u_{x}\right)_{x}, U\right)+\left(\partial_{0 t}^{\alpha}\left(x^{m} \eta u_{x}\right)_{x}, U\right) \\
& +\left(r u_{x}, x^{m} U\right)-\left(\int_{0}^{s} q u d s, x^{m} U\right)+\left(f, x^{m} U\right) .
\end{aligned}
$$

Taking into consideration (2.2)-(2.7), after some simple transformations by (5.1) we find:

$$
\begin{aligned}
& \frac{1}{2} \partial_{0 t}^{\alpha}\left\|x^{\frac{m}{2}} u\right\|_{0}^{2}+\frac{1}{2} \int_{0}^{l}(k+\eta(x)) \partial_{0 t}^{\alpha}\left(x^{\frac{m}{2}} u_{x}\right)^{2} d x+c_{0}\left\|x^{\frac{m}{2}} u_{x}\right\|_{0}^{2}+\frac{1}{2}\left\|\partial_{0 t}^{\alpha} x^{\frac{m}{2}} u\right\|_{0}^{2} \\
& c_{0}\left\|\partial_{0 t}^{\alpha} x^{\frac{m}{2}} u_{x}\right\|_{0}^{2} \leqslant\left. x^{m} U \Pi(x, t)\right|_{0} ^{l}+M_{7}\left(\left\|x^{\frac{m}{2}} u\right\|_{0}^{2}+\left\|x^{\frac{m}{2}} u_{x}\right\|_{0}^{2}\right)+M_{8}\left\|x^{\frac{m}{2}} f\right\|_{0}^{2} .
\end{aligned}
$$

We estimate the first term in the right hand side in (5.2):

$$
\begin{aligned}
\left.x^{m} U \Pi(x, t)\right|_{0} ^{l}= & l^{m}\left(u(l, t)+\partial_{0 t}^{\alpha} u(l, t)\right) \Pi(l, t) \\
= & l^{m}\left(u(l, t)+\partial_{0 t}^{\alpha} u(l, t)\right)\left(\mu(t)-\beta_{1}(t) u(l, t)-\beta_{2}(t) \partial_{0 t}^{\alpha} u(l, t)\right) \\
= & l^{m} u(l, t) \mu(t)+l^{m} \mu(t) \partial_{0 t}^{\alpha} u(l, t)-l^{m} u^{2}(l, t) \beta_{1}(t)-l^{m} \beta_{1}(t) u(l, t) \partial_{0 t}^{\alpha} u(l, t) \\
& -l^{m} \beta_{2}(t) u(l, t) \partial_{0 t}^{\alpha} u(l, t)-l^{m} \beta_{2}(t)\left(\partial_{0 t}^{\alpha} u(l, t)\right)^{2} \\
\leqslant & -l^{m} \beta_{2}(t)\left(\partial_{0 t}^{\alpha} u(l, t)\right)^{2}-\frac{l^{m} \beta_{2}(t)}{2} \partial_{0 t}^{\alpha} u^{2}(l, t) \\
& +\varepsilon\left(\partial_{0 t}^{\alpha} u(l, t)\right)^{2}+M_{9}\left(\left\|x^{\frac{m}{2}} u\right\|_{0}^{2}+\left\|x^{\frac{m}{2}} u_{x}\right\|_{0}^{2}\right)+M_{10} \mu^{2}(t) \\
\leqslant & -\frac{l^{m} \beta_{2}(t)}{2}\left(\partial_{0 t}^{\alpha} u(l, t)\right)^{2}-\frac{l^{m} \beta_{2}(t)}{2} \partial_{0 t}^{\alpha} u^{2}(l, t) \\
& +M_{9}\left(\left\|x^{\frac{m}{2}} u\right\|_{0}^{2}+\left\|x^{\frac{m}{2}} u_{x}\right\|_{0}^{2}\right)+M_{10} \mu^{2}(t) .
\end{aligned}
$$

In view of (5.3), by (5.2) we find:

$$
\begin{gathered}
\partial_{0 t}^{\alpha}\left\|x^{\frac{m}{2}} u\right\|_{0}^{2}+\int_{0}^{l}(k+\eta(x)) \partial_{0 t}^{\alpha}\left(x^{\frac{m}{2}} u_{x}\right)^{2} d x+\left\|x^{\frac{m}{2}} u_{x}\right\|_{0}^{2}+\left\|\partial_{0 t}^{\alpha} x^{\frac{m}{2}} u\right\|_{0}^{2}+\left\|\partial_{0 t}^{\alpha} x^{\frac{m}{2}} u_{x}\right\|_{0}^{2} \\
\leqslant M_{11}\left\|x^{\frac{m}{2}} u\right\|_{W_{2}^{1}(0, l)}^{2}+M_{12}\left(\left\|x^{\frac{m}{2}} f\right\|_{0}^{2}+\mu_{2}(t)\right)
\end{gathered}
$$

where $\left\|x^{\frac{m}{2}} u\right\|_{W_{2}^{1}(0, l)}^{2}=\left\|x^{\frac{m}{2}} u\right\|_{0}^{2}+\left\|x^{\frac{m}{2}} u_{x}\right\|_{0}^{2}$. Applying the operator of the fractional integration $D_{0 t}^{-\alpha}$ to the both sides of inequality (5.4), we find:

$$
\begin{aligned}
\left\|x^{\frac{m}{2}} u\right\|_{W_{2}^{1}(0, l)}^{2} & +D_{0 t}^{-\alpha}\left(\left\|x^{\frac{m}{2}} u_{x}\right\|_{0}^{2}+\left\|\partial_{0 t}^{\alpha} x^{\frac{m}{2}} u\right\|_{0}^{2}+\left\|\partial_{0 t}^{\alpha} x^{\frac{m}{2}} u_{x}\right\|_{0}^{2}\right) \\
& \leqslant M_{13} D_{0 t}^{-\alpha}\left\|x^{\frac{m}{2}} u\right\|_{W_{2}^{1}(0, l)}^{2}+M_{15}\left(D_{0 t}^{-\alpha}\left(\left\|x^{\frac{m}{2}} f\right\|_{0}^{2}+\mu_{2}^{2}(t)\right)+\left\|x^{\frac{m}{2}} u_{0}\right\|_{W_{2}^{1}(0, l)}^{2}\right) .
\end{aligned}
$$


By Lemma 2 and (5.5) we obtain the sought apriori estimate:

$$
\begin{aligned}
\left\|x^{\frac{m}{2}} u\right\|_{W_{2}^{1}(0, l)}^{2} & +D_{0 t}^{-\alpha}\left(\left\|x^{\frac{m}{2}} u_{x}\right\|_{0}^{2}+\left\|\partial_{0 t}^{\alpha} x^{\frac{m}{2}} u\right\|_{0}^{2}+\left\|\partial_{0 t}^{\alpha} x^{\frac{m}{2}} u_{x}\right\|_{0}^{2}\right) \\
& \leqslant M\left(D_{0 t}^{-\alpha}\left(\left\|x^{\frac{m}{2}} f\right\|_{0}^{2}+\mu_{2}^{2}(t)\right)+\left\|x^{\frac{m}{2}} u_{0}(x)\right\|_{W_{2}^{1}(0, l)}^{2}\right),
\end{aligned}
$$

where $M$ is a positive constant depending only on the data of problem (4.1)-(4.4), and

$$
D_{0 t}^{-\alpha} u=\frac{1}{\Gamma(\alpha)} \int_{0}^{t} \frac{u d \tau}{(t-\tau)^{1-\alpha}}
$$

is the fractional Riemann-Liouville integral of order $\alpha, 0<\alpha<1$.

Theorem 3. If $k(x, t) \in C^{1,0}\left(\bar{Q}_{T}\right), \eta(x) \in C^{1}[0, l], r(x, t), q(x, t), f(x, t) \in C\left(\bar{Q}_{T}\right), u(x, t) \in$ $C^{2,0}\left(Q_{T}\right) \cap C^{1,0}\left(\bar{Q}_{T}\right), \partial_{0 t}^{\alpha} u(x, t) \in C\left(\bar{Q}_{T}\right)$ and conditions (1.5) are satisfied, then the solution of problem (4.1)-(4.4) obeys apriori estimate (5.6).

Apriori estimate (5.6) implies the uniqueness of the solution and the stability with respect to the initial data and the right hand side in the sense of the norm:

$$
\left\|x^{\frac{m}{2}} u\right\|_{1}^{2}=\left\|x^{\frac{m}{2}} u\right\|_{W_{2}^{1}(0, l)}^{2}+D_{0 t}^{-\alpha}\left(\left\|x^{\frac{m}{2}} u_{x}\right\|_{0}^{2}+\left\|\partial_{0 t}^{\alpha} x^{\frac{m}{2}} u\right\|_{0}^{2}+\left\|\partial_{0 t}^{\alpha} x^{\frac{m}{2}} u_{x}\right\|_{0}^{2}\right) .
$$

\section{Stability And CONVERGence of Difference SCHEME}

On the uniform grid $\bar{\omega}_{h \tau}$, we consider a difference scheme for differential problem (4.1)-(4.4) of approximation order $O\left(h^{2}+\tau^{2}\right)$ :

$$
\begin{gathered}
\bar{\varkappa}_{0 t_{j+\sigma}}^{\alpha} y=\frac{\varkappa}{x_{i}^{m}}\left(x_{i-0.5}^{m} a^{j} y_{\bar{x}}^{(\sigma)}\right)_{x}+\frac{1}{x_{i}^{m}} \Delta_{0 t_{j+\sigma}}^{\alpha}\left(x_{i-0.5}^{m} \gamma_{i} y_{\bar{x}, i}\right)_{x}+\frac{b^{-j}}{x_{i}^{m}}\left(x_{i-0.5}^{m} a_{i}^{j} y_{\bar{x}, i}^{(\sigma)}\right) \\
+\frac{b^{+j}}{x_{i}^{m}}\left(x_{i+0.5}^{m} a_{i+1}^{j} y_{x, i}^{(\sigma)}\right)-\sum_{s=0}^{i} d_{s}^{j} y_{s}^{(\sigma)} \hbar+\varphi_{i}^{j}, \quad(x, t) \in \omega_{h, \tau}, \\
\varkappa_{0} a_{1} y_{x, 0}^{(\sigma)}+\Delta_{0 t_{j+\sigma}}^{\alpha}\left(\gamma_{1} y_{\bar{x}, 0}\right)=\frac{0.5 h}{m+1}\left(\Delta_{0 t_{j+\sigma}}^{\alpha} y_{0}+0.5 h d_{0}^{j} y_{0}^{(\sigma)}\right)-\tilde{\mu}_{1}, \\
-\varkappa_{N} a_{N} y_{\bar{x}, N}^{(\sigma)}-\Delta_{0 t_{j+\sigma}}^{\alpha}\left(\gamma_{N} y_{\bar{x}, N}\right)=\tilde{\beta}_{1} y_{N}^{(\sigma)}+0.5 h \sum_{s=0}^{N} d_{s}^{j} y_{s}^{(\sigma)} \hbar+\tilde{\beta}_{2} \Delta_{0 t_{j+\sigma}}^{\alpha} y_{N}-\tilde{\mu}_{2}, \\
y(x, 0)=u_{0}(x), \quad x \in \bar{\omega}_{h},
\end{gathered}
$$

where

$$
\begin{aligned}
& \tilde{\beta}_{1}=\tilde{\varkappa} \beta_{1}\left(t_{j+\sigma}\right), \quad \tilde{\beta}_{2}=\tilde{\varkappa} \beta_{2}+0.5 h, \quad \tilde{\mu}_{1}=\frac{0.5 h}{m+1} \varphi_{0}^{j}, \quad \tilde{\mu}_{2}=\tilde{\varkappa} \mu\left(t_{j+\sigma}\right)+0.5 h \varphi_{N}^{j}, \\
& \varkappa_{0}=\frac{1}{1+\frac{0.5 h\left|r_{0}\right|}{(m+1) k_{0.5}^{j+\sigma}}} \quad \text { if } \quad r_{0}^{j+\sigma} \leqslant 0, \quad \varkappa_{N}=\frac{1}{1+\frac{0.5 h\left|r_{N}^{j+\sigma}\right|}{k_{N-0.5}}} \quad \text { if } \quad r_{N}^{j+\sigma} \geqslant 0, \\
& r=r^{+}+r^{-}, \quad|r|=r^{+}-r^{-}, \quad r^{+}=0.5(r+|r|) \geqslant 0, \quad r^{-}=0.5(r-|r|) \leqslant 0, \\
& a_{i}^{j}=k\left(x_{i-0.5}, t^{j+\sigma}\right), \quad \gamma_{i}=\eta\left(x_{i-0.5}\right), \quad b_{i}^{ \pm j}=\frac{\bar{\varkappa}_{i} r_{i}^{ \pm j+\sigma}}{k_{i}^{j+\sigma},} \quad \bar{\varkappa}_{i}=1+\frac{m(m-1) h^{2}}{24 x_{i}^{2}}, \\
& d_{i}^{j}= \begin{cases}\bar{\varkappa}_{i} q_{i}^{j+\sigma}, & i=\overline{1, N-1}, \quad \varphi_{i}^{j}= \begin{cases}\bar{\varkappa}_{i} f_{i}^{j+\sigma}, & i=1, N-1, \\
f_{i}^{j+\sigma}, & i=0, N,\end{cases} \end{cases}
\end{aligned}
$$




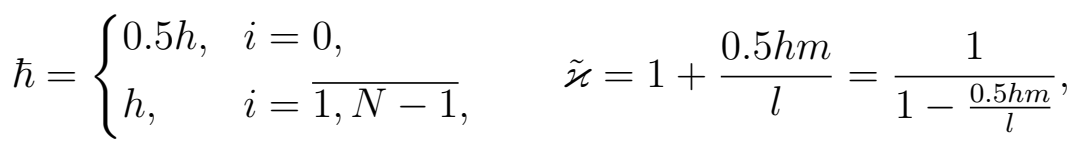

$$
\begin{aligned}
& \varkappa_{i}=\frac{1}{1+R_{i}}, \quad R_{i}=\frac{0.5 h\left|r_{i}\right| \bar{\varkappa}_{i}}{k_{i-0.5}} .
\end{aligned}
$$

We are going to obtain an apriori estimate by the method of energy inequalities. In order to do this, we rewrite (6.1)-(6.4) in the operator form:

$$
\begin{aligned}
& \overline{\bar{\varkappa}} \Delta_{t_{j+\sigma}}^{\alpha} y=\bar{\Lambda}\left(t_{j+\sigma}\right) y^{(\sigma)}+\bar{\delta} y+\bar{\Phi}, \\
& y(x, 0)=u_{0}(x)
\end{aligned}
$$

where

$$
\begin{aligned}
& \overline{\bar{\varkappa}}=\left\{\begin{array}{ll}
\bar{\varkappa}_{i}, & x \in \omega_{h}, \\
1, & x=0, l,
\end{array} \quad \bar{\varkappa}_{i}=1+\frac{m(m-1) h^{2}}{24 x_{i}^{2}},\right. \\
& \bar{\delta} y= \begin{cases}\delta y_{i}=\frac{1}{x_{i}^{m}} \Delta_{0 t_{j+\sigma}}^{\alpha}\left(x_{i-0.5}^{m} \gamma_{i} y_{\bar{x}, i}\right)_{x}, & (x, t) \in \omega_{h, \tau} \\
\delta^{-} y_{0}=\frac{m+1}{0.5 h} \Delta_{0 t_{j+\sigma}}^{\alpha}\left(\gamma_{1} y_{x, 0}\right), & x=0 \\
\delta^{+} y_{N}=-\frac{2}{h}\left(\Delta_{0 t_{j+\sigma}}^{\alpha}\left(\gamma_{N} y_{\bar{x}, N}\right)+\tilde{\varkappa} \beta_{2} \Delta_{0 t_{j+\sigma}}^{\alpha} y_{N}\right), & x=l .\end{cases} \\
& \bar{\Lambda}\left(t^{j+\sigma}\right) y^{\sigma}=\left\{\begin{array}{cc}
\tilde{\Lambda}\left(t^{j+\sigma}\right) y_{i}^{(\sigma)}=\frac{\varkappa_{i}}{x_{i}^{m}}\left(x_{i-0.5}^{m} a_{i}^{j} y_{\bar{x}, i}^{(\sigma)}\right)_{x}+\frac{b^{-j}}{x_{i}^{m}}\left(x_{i-0.5}^{m} a_{i}^{j} y_{\bar{x}}^{(\sigma)}\right) & \\
\quad+\frac{b^{+j}}{x_{i}^{m}}\left(x_{i+0.5}^{m} a_{i+1}^{j} y_{x}^{(\sigma)}\right)-\sum_{s=0}^{i} d_{s}^{j} y_{s}^{(\sigma)} \hbar, & x=0, \\
\Lambda^{-} y_{0}^{(\sigma)}=\frac{m+1}{0.5 h}\left(\varkappa_{0} a_{1} y_{x, 0}^{(\sigma)}-0.5 h d_{0}^{j} y_{0}^{(\sigma)}\right), & x=l, \\
\Lambda^{+} y_{N}^{(\sigma)}=-\frac{2}{h}\left(\varkappa_{N} a_{N} y_{x, N}^{(\sigma)}+\tilde{\beta}_{1} y_{N}^{(\sigma)}+0.5 h \sum_{s=0}^{N} d_{s}^{j} y_{s}^{(\sigma)} \hbar\right), &
\end{array}\right. \\
& \bar{\Phi}= \begin{cases}\varphi=\varphi_{i}, & (x, t) \in \omega_{h \tau}, \\
\varphi^{-}=\frac{m+1}{0.5 h} \tilde{\mu}_{1}, & x=0, \\
\varphi^{+}=\frac{1}{0.5 h} \tilde{\mu}_{2}, & x=l .\end{cases}
\end{aligned}
$$

We calculate the scalar product of (6.5) with $x^{m} \bar{y}=x^{m} y^{(\sigma)}+x^{m} \Delta_{0 t_{j+\sigma}}^{\alpha} y$ :

$$
\left(\overline{\bar{\varkappa}} \Delta_{0 t_{j+\sigma}}^{\alpha} y, x^{m} \bar{y}\right)=\left(\bar{\Lambda}\left(t_{j+\sigma}\right) y^{(\sigma)}, x^{m} \bar{y}\right]+\left(\bar{\delta} y, x^{m} \bar{y}\right]+\left(\bar{\Phi}, x^{m} \bar{y}\right],
$$

where

$$
\left.(u, v]=\sum_{i=1}^{N} u_{i} v_{i} \hbar, \quad \| u\right]\left.\right|_{0} ^{2}=\sum_{i=1}^{N} u_{i}^{2} \hbar, \quad \hbar= \begin{cases}0.5 h, & i=0, N \\ h, & i \neq 0, N .\end{cases}
$$

We transform the sums in identity (6.7) employing the Cauchy inequality with $\varepsilon$ :

$$
\begin{aligned}
\left(\overline{\bar{\varkappa}} \Delta_{0 t_{j+\sigma}}^{\alpha} y, x^{m} \bar{y}\right) & =\left(\overline{\bar{\varkappa}} \Delta_{0 t_{j+\sigma}}^{\alpha} y, x^{m} y^{(\sigma)}\right]+\left(\overline{\bar{\varkappa}} \Delta_{0 t_{j+\sigma}}^{\alpha} y, x^{m} \Delta_{0 t_{j+\sigma}}^{\alpha} y\right] \\
& \geqslant\left(\frac{\overline{\bar{\varkappa}}}{2}, \Delta_{0 t_{j+\sigma}}^{\alpha}\left(x^{\frac{m}{2}} y\right)^{2}\right]+\left(\overline{\bar{\varkappa}},\left(\Delta_{0 t_{j+\sigma}}^{\alpha}\left(x^{\frac{m}{2}} y\right)\right)^{2}\right],
\end{aligned}
$$




$$
\begin{aligned}
\left(\bar{\Lambda}\left(t^{j+\sigma}\right) y^{(\sigma)},\right. & \left.x^{m} \bar{y}\right]=\left(\tilde{\Lambda} y^{(\sigma)}, x^{m} \bar{y}\right)+0.5 h \Lambda^{+} y_{N}^{(\sigma)} x_{N}^{m} \bar{y}_{N} \\
= & \left(\varkappa\left(x_{i-0.5}^{m} a_{i} y_{\bar{x}}^{(\sigma)}\right)_{x}, \bar{y}\right)+\left(b^{-}\left(x_{i-0.5}^{m} a_{i}^{j} y_{\bar{x}}^{(\sigma)}\right), \bar{y}\right)+\left(b^{+}\left(x_{i+0.5}^{m} a_{i+1}^{j} y_{x}^{(\sigma)}\right), \bar{y}\right) \\
& -\left(\sum_{s=0}^{i} d_{s}^{j} y_{s}^{(\sigma)} \hbar, x_{i}^{m} \bar{y}\right)-x_{N}^{m} \bar{y}_{N}\left(\varkappa_{N} a_{N} y_{\bar{x}, N}^{(\sigma)}+\tilde{\beta}_{1} y_{N}^{(\sigma)}+0.5 h \sum_{s=0}^{N} d_{s}^{j} y_{s}^{(\sigma)} \hbar\right) \\
= & -\left(x_{i-0.5}^{m} a_{i} y_{\bar{x}}^{(\sigma)},(\varkappa \bar{y})_{\bar{x}}\right]+\left(b^{-}\left(\bar{x}^{m} a_{i} y_{\bar{x}}^{(\sigma)}\right), \bar{y}\right)+\left(b^{+} x_{i+0.5}^{m} a^{(+1)}, y_{x}^{(\sigma)} \bar{y}\right) \\
& -\left(\sum_{s=0}^{i} d_{s}^{j} y_{s}^{(\sigma)} \hbar, x^{m} \bar{y}\right)-\tilde{\beta}_{1} x_{N}^{m} y_{N}^{(\sigma)} \bar{y}_{N}-x_{N}^{m} 0.5 h \bar{y}_{N} \sum_{s=0}^{N} d_{s}^{j} y_{s}^{(\sigma)} \hbar \\
& +\left(\bar{x}_{N}^{m}-x_{N}^{m}\right) \bar{y}_{N} \varkappa_{N} a_{N} y_{\bar{x}, N}^{(\sigma)}-x_{0.5}^{m} \varkappa_{0} a_{1} y_{x, 0}^{(\sigma)} \bar{y}_{0} .
\end{aligned}
$$

Let us transform the terms in the right hand of the above identity:

$$
\begin{aligned}
& -\left(x_{i-0.5}^{m} a_{i} y_{\bar{x}}^{(\sigma)},(\varkappa \bar{y})_{\bar{x}}\right]=-\left(x_{i-0.5}^{m} a_{i} y_{\bar{x}}^{(\sigma)}, \varkappa_{\bar{x}} \bar{y}+\varkappa^{(-1)} \bar{y}_{\bar{x}}\right] \\
& =-\left(\bar{x}^{m} a y_{\bar{x}}^{(\sigma)}, \varkappa_{\bar{x}} y^{(\sigma)}\right]-\left(\bar{x}^{m} a y_{\bar{x}}^{(\sigma)}, \varkappa_{\bar{x}} \Delta_{0 t_{j+\sigma}}^{\alpha} y\right] \\
& -\left(\bar{x}^{m} a y_{\bar{x}}^{(\sigma)}, \varkappa^{(-1)} y_{\bar{x}}^{(\sigma)}\right]-\left(\bar{x}^{m} a y_{\bar{x}}^{(\sigma)}, \varkappa^{(-1)} \Delta_{0 t_{j+\sigma}}^{\alpha} y_{\bar{x}}\right] \\
& \left.\left.\left.\leqslant \varepsilon \| \Delta_{0 t_{j+\sigma}}^{\alpha} x^{\frac{m}{2}} y\right]\left.\right|_{0} ^{2}+\left.M_{1}^{\varepsilon}\left(\| x^{\frac{m}{2}} y^{(\sigma)}\right]\right|_{0} ^{2}+\| \bar{x}^{\frac{m}{2}} y_{\bar{x}}^{(\sigma)}\right]\left.\right|_{0} ^{2}\right) \\
& -\frac{1}{1+h M_{2}}\left(\bar{x}^{m} a \varkappa,\left(y_{\bar{x}}^{(\sigma)}\right)^{2}\right]-\frac{1}{2\left(1+h M_{2}\right)}\left(\bar{x}^{m} a \varkappa, \Delta_{0 t_{j+\sigma}}^{\alpha} y_{\bar{x}}^{2}\right] \\
& \left.\left.\left.\leqslant \varepsilon \| \Delta_{0 t_{j+\sigma}}^{\alpha} x^{\frac{m}{2}} y\right]\left.\right|_{0} ^{2}+\left.M_{1}^{\varepsilon}\left(\| x^{\frac{m}{2}} y^{(\sigma)}\right]\right|_{0} ^{2}+\| \bar{x}^{\frac{m}{2}} y_{\bar{x}}^{(\sigma)}\right]\left.\right|_{0} ^{2}\right) \\
& \left.\left.-M_{3} \| \bar{x}^{\frac{m}{2}} y_{\bar{x}}^{(\sigma)}\right]\left.\right|_{0} ^{2}-M_{4} \Delta_{0 t_{j+\sigma}}^{\alpha} \| \bar{x}^{\frac{m}{2}} y_{\bar{x}}\right]\left.\right|_{0} ^{2}, \\
& \left(b^{-}\left(\bar{x}^{m} a_{i}^{j} y_{\bar{x}}^{(\sigma)}\right), \bar{y}\right)+\left(b^{+} x_{i+0.5}^{m} a^{(+1)}, y_{x}^{(\sigma)} \bar{y}\right) \\
& =\left(b^{-} \bar{x}^{m} a y_{\bar{x}}^{(\sigma)}, y^{(\sigma)}\right)+\left(b^{-} \bar{x}^{m} a y_{\bar{x}}^{(\sigma)}, \Delta_{0 t_{j+\sigma}}^{\alpha} y\right) \\
& +\left(b^{+} x_{i+0.5}^{m} a^{(+1)} y_{x}^{(\sigma)}, y^{(\sigma)}\right)+\left(b^{+} x_{i+0.5}^{m} a^{(+1)} y_{x}^{(\sigma)}, \Delta_{0 t_{j+\sigma}}^{\alpha} y\right) \\
& \left.\left.\left.\leqslant \varepsilon \| \Delta_{0 t_{j+\sigma}}^{\alpha} x^{\frac{m}{2}} y\right]\left.\right|_{0} ^{2}+\left.M_{5}^{\varepsilon}\left(\| x^{\frac{m}{2}} y^{(\sigma)}\right]\right|_{0} ^{2}+\| \bar{x}^{\frac{m}{2}} y_{\bar{x}}^{(\sigma)}\right]\left.\right|_{0} ^{2}\right) \text {, } \\
& -\left(\sum_{s=0}^{i} d_{s}^{j} y_{s}^{(\sigma)} \hbar, x^{m} \bar{y}\right)-\tilde{\beta}_{1} x_{N}^{m} y_{N}^{(\sigma)} \bar{y}_{N}-x_{N}^{m} 0.5 h \bar{y}_{N} \sum_{s=0}^{N} d_{s}^{j} y_{s}^{(\sigma)} \hbar \\
& =-\left(\sum_{s=0}^{i} d_{s}^{j} y_{s}^{(\sigma)} \hbar, x^{m} \bar{y}\right]-\tilde{\beta}_{1} x_{N}^{m} y_{N}^{(\sigma)} \bar{y}_{N} \\
& \leqslant-\left(\sum_{s=0}^{i} d_{s}^{j} y_{s}^{(\sigma)} \hbar, x^{m} y^{(\sigma)}\right]-\left(\sum_{s=0}^{i} d_{s}^{j} y_{s}^{(\sigma)} \hbar, x^{m} \Delta_{0 t_{j+\sigma}}^{\alpha} y\right] \\
& -\tilde{\beta}_{1} x_{N}^{m}\left(y_{N}^{(\sigma)}\right)^{2}-\tilde{\beta}_{1} x_{N}^{m} y_{N}^{(\sigma)} \Delta_{0 t_{j+\sigma}}^{\alpha} y_{N} \\
& \left.\leqslant \varepsilon_{1} \| \Delta_{0 t_{j+\sigma}}^{\alpha} x^{\frac{m}{2}} y\right]\left.\right|_{0} ^{2}+\varepsilon_{2}\left(\Delta_{0 t_{j+\sigma}}^{\alpha} y_{N}\right)^{2} \\
& \left.\left.+\left.M_{6}^{\varepsilon_{1}, \varepsilon_{2}}\left(\| x^{\frac{m}{2}} y^{(\sigma)}\right]\right|_{0} ^{2}+\| \bar{x}^{\frac{m}{2}} y_{\bar{x}}^{(\sigma)}\right]\left.\right|_{0} ^{2}\right) .
\end{aligned}
$$


Taking into consideration (6.9)-(6.12), we obtain:

$$
\begin{aligned}
\left(\bar{\Lambda}\left(t^{j+\sigma}\right) y^{(\sigma)}, x^{m} \bar{y}\right] \leqslant & \left.\varepsilon_{1} \| \Delta_{0 t_{j+\sigma}}^{\alpha} x^{\frac{m}{2}} y\right]\left.\right|_{0} ^{2}+\varepsilon_{2}\left(\Delta_{0 t_{j+\sigma}}^{\alpha} y_{N}\right)^{2} \\
& \left.\left.+\left.M_{7}^{\varepsilon_{1}, \varepsilon_{2}}\left(\| x^{\frac{m}{2}} y^{(\sigma)}\right]\right|_{0} ^{2}+\| \bar{x}^{\frac{m}{2}} y_{\bar{x}}^{(\sigma)}\right]\left.\right|_{0} ^{2}\right) \\
& \left.\left.-M_{3} \| \bar{x}^{\frac{m}{2}} y_{\bar{x}}^{(\sigma)}\right]\left.\right|_{0} ^{2}-M_{4} \Delta_{0 t_{j+\sigma}}^{\alpha} \| \bar{x}^{\frac{m}{2}} y_{\bar{x}}\right]\left.\right|_{0} ^{2} \\
& +\left(\bar{x}_{N}^{m}-x_{N}^{m}\right) \bar{y}_{N} \varkappa_{N} a_{N} y_{\bar{x}, N}^{(\sigma)}-x_{0.5}^{m} \varkappa_{0} a_{1} y_{x, 0}^{(\sigma)} \bar{y}_{0}, \\
\left(\bar{\delta} y, x^{m} \bar{y}\right]= & \left(\delta y, \bar{y} x^{m}\right)+0.5 h x_{N}^{m} \bar{y}_{N} \delta^{+} y_{N} \\
= & \left(\Delta_{0 t_{j+\sigma}}^{\alpha}\left(\bar{x}^{m} \gamma y_{\bar{x}}\right)_{x}, \bar{y}\right)+0.5 h x_{N}^{m} \bar{y}_{N} \delta^{+} y_{N} \\
= & -\left(\Delta_{0 t_{j+\sigma}}^{\alpha} \bar{x}^{m} \gamma y_{\bar{x}}, \bar{y}_{\bar{x}}\right]+\bar{x}_{N}^{m} \gamma_{N} \bar{y}_{N} \Delta_{0 t_{j+\sigma}}^{\alpha} y_{\bar{x}, N} \\
& -x_{N}^{m} \bar{y}_{N} \gamma_{N} \Delta_{0 t_{j+\sigma}}^{\alpha} y_{\bar{x}, N}-\tilde{\varkappa} \beta_{2} x_{N}^{m} \bar{y}_{N} \Delta_{0 t_{j+\sigma}}^{\alpha} y_{N}-x_{0.5}^{m} \bar{y}_{0} \Delta_{0 t_{j+\sigma}}^{\alpha}\left(\gamma_{1} y_{x, 0}\right) .
\end{aligned}
$$

We transform the terms in the right hand side in (6.14):

$$
\begin{aligned}
-\left(\Delta_{0 t_{j+\sigma}}^{\alpha} \bar{x}^{m} \gamma y_{\bar{x}}, \bar{y}_{\bar{x}}\right] & =-\left(\Delta_{0 t_{j+\sigma}}^{\alpha} \bar{x}^{m} \gamma y_{\bar{x}}, y_{\bar{x}}^{(\sigma)}\right]-\left(\Delta_{0 t_{j+\sigma}}^{\alpha} \bar{x}^{m} \gamma y_{\bar{x}}, \Delta_{0 t_{j+\sigma}}^{\alpha} y_{\bar{x}}\right] \\
& \left.\left.\leqslant-\frac{c_{0}}{2} \Delta_{0 t_{j+\sigma}}^{\alpha} \| \bar{x}^{\frac{m}{2}} y_{x}\right]\left.\right|_{0} ^{2}-c_{0} \| \Delta_{0 t_{j+\sigma}}^{\alpha} \bar{x}^{\frac{m}{2}} y_{\bar{x}}\right]\left.\right|_{0} ^{2}, \\
-\tilde{\varkappa} \beta_{2} x_{N}^{m} \bar{y}_{N} \Delta_{0 t_{j+\sigma}}^{\alpha} y_{N} & =-\tilde{\varkappa} \beta_{2} x_{N}^{m} y_{N}^{(\sigma)} \Delta_{0 t_{j+\sigma}}^{\alpha} y_{N}-\tilde{\varkappa} \beta_{2} x_{N}^{m}\left(\Delta_{0 t_{j+\sigma}}^{\alpha} y_{N}\right)^{2} .
\end{aligned}
$$

By (6.15) and (6.16) we obtain:

$$
\begin{aligned}
\left(\bar{\delta} y, x^{m} \bar{y}\right] \leqslant & \left.\left.-\frac{c_{0}}{2} \Delta_{0 t_{j+\sigma}}^{\alpha} \| \bar{x}^{\frac{m}{2}} y_{\bar{x}}\right]\left.\right|_{0} ^{2}-c_{0} \| \Delta_{0 t_{j+\sigma}}^{\alpha} \bar{x}^{\frac{m}{2}} y_{\bar{x}}\right]\left.\right|_{0} ^{2}+\left(\bar{x}_{N}^{m}-x_{N}^{m}\right) \bar{y}_{N} \Delta_{0 t_{j+\sigma}}^{\alpha}\left(\gamma_{N} y_{\bar{x}, N}\right) \\
& -x_{0.5}^{m} \bar{y}_{0} \Delta_{0 t_{j+\sigma}}^{\alpha}\left(\gamma_{1} y_{x, 0}\right)-\tilde{\varkappa} \frac{\beta_{2}}{2} x_{N}^{m} \Delta_{0 t_{j+\sigma}}^{\alpha}\left(y_{N}\right)^{2}-\tilde{\varkappa} x_{N}^{m} \beta_{2}\left(\Delta_{0 t_{j+\sigma}}^{\alpha} y_{N}\right)^{2} \\
\left(\bar{\Phi}, x^{m} \bar{y}\right]= & \left(\varphi, x^{m} \bar{y}\right)+0.5 h x_{N}^{m} \bar{y}_{N} \varphi^{+}=\left(\varphi, x^{m} \bar{y}\right)+x_{N}^{m} \tilde{\mu}^{2} \bar{y}_{N} \\
= & \left(\varphi, x^{m} y^{(\sigma)}\right)+\left(\varphi, x^{m} \Delta_{0 t_{j+\sigma}}^{\alpha} y\right)+x_{N}^{m} \tilde{\mu}_{2} \bar{y}_{N} \\
\leqslant & \left.\varepsilon_{1} \| \Delta_{0 t_{j+\sigma}}^{\alpha} x^{\frac{m}{2}} y\right]\left.\right|_{0} ^{2}+\left.M_{8}^{\varepsilon_{1}}\left(\| x^{\frac{m}{2}} y^{(\sigma)}\right]\right|_{0} ^{2} \\
& \left.\left.+\| \bar{x}^{\frac{m}{2}} y_{\bar{x}}^{(\sigma)}\right]\left.\right|_{0} ^{2}\right)+M_{9}^{\varepsilon_{1}}\left\|x^{\frac{m}{2}} \varphi\right\|_{0}^{2}+x_{N}^{m} \tilde{\mu}_{2} \bar{y}_{N} .
\end{aligned}
$$

Bearing in mind (6.8)-(6.18), by (6.7) we obtain:

$$
\begin{aligned}
\left(\frac{\overline{\bar{\varkappa}}}{2}, \Delta_{0 t_{j+\sigma}}^{\alpha}\left(x^{\frac{m}{2}} y\right)^{2}\right] & \left.\left.+M_{10} \Delta_{0 t_{j+\sigma}}^{\alpha} \| \bar{x}^{\frac{m}{2}} y_{\bar{x}}\right]\left.\right|_{0} ^{2}+M_{3} \| \bar{x}^{\frac{m}{2}} y_{\bar{x}}^{(\sigma)}\right]\left.\right|_{0} ^{2}+\left(\overline{\bar{\varkappa}},\left(\Delta_{0 t_{j+\sigma}}^{\alpha}\left(x^{\frac{m}{2}} y\right)\right)^{2}\right] \\
& \left.+c_{0} \| \Delta_{0 t_{j+\sigma}}^{\alpha} \bar{x}^{\frac{m}{2}} y_{\bar{x}}\right]\left.\right|_{0} ^{2}+\frac{\beta_{2}}{2} x_{N}^{m} \Delta_{0 t_{j+\sigma}}^{\alpha}\left(y_{N}\right)^{2}+\beta_{2} x_{N}^{m}\left(\Delta_{0 t_{j+\sigma}}^{\alpha} y_{N}\right)^{2} \\
\leqslant & \left.\varepsilon_{1} \| \Delta_{0 t_{j+\sigma}}^{\alpha} x^{\frac{m}{2}} y\right]\left.\right|_{0} ^{2}+\varepsilon_{2}\left(\Delta_{0 t_{j+\sigma}}^{\alpha} y_{N}\right)^{2} \\
& +\left(\bar{x}_{N}^{m}-x_{N}^{m}\right)\left(x_{N} a_{N} y_{\bar{x}, N}^{(\sigma)}+\Delta_{0 t_{j+\sigma}}^{\alpha}\left(\gamma_{N} y_{\bar{x}, N}\right)\right) \bar{y}_{N} \\
& -x_{0.5}^{m} \bar{y}_{0}\left(\varkappa_{0} a_{1} y_{x, 0}^{(\sigma)}+\Delta_{0 t_{j+\sigma}}^{\alpha}\left(\gamma_{1} y_{x, 0}\right)\right)+M_{8}\left(\varepsilon_{1}\right)\left\|x^{\frac{m}{2}} \varphi\right\|_{0}^{2}+x_{N}^{m} \tilde{\mu}_{2} \bar{y}_{N} \\
& \left.\left.+\left.M_{11}\left(\varepsilon_{1}, \varepsilon_{2}\right)\left(\| x^{\frac{m}{2}} y^{(\sigma)}\right]\right|_{0} ^{2}+\| \bar{x}^{\frac{m}{2}} y_{\bar{x}}^{(\sigma)}\right]\left.\right|_{0} ^{2}\right) .
\end{aligned}
$$


The third, fourth and sixth term in the right hand in the above identity we get:

$$
\begin{aligned}
&\left(\bar{x}_{N}^{m}-x_{N}^{m}\right)\left(\varkappa_{N} a_{N} y_{\bar{x}, N}^{(\sigma)}+\Delta_{0 t_{j+\sigma}}^{\alpha}\left(\gamma_{N} y_{\bar{x}, N}\right)\right) \bar{y}_{N}-x_{0.5}^{m} \bar{y}_{0}\left(\varkappa_{0} a_{1} y_{x, 0}^{(\sigma)}+\Delta_{0 t_{j+\sigma}}^{\alpha}\left(\gamma_{1} y_{x, 0}\right)\right) \\
&+x_{N}^{m} \tilde{\mu}_{2} \bar{y}_{N}= x_{0.5}^{m} \bar{y}_{0}\left(\tilde{\mu}_{1}-\frac{0.5 h}{m+1}\left(\Delta_{0 t_{j+\sigma}}^{\alpha} y_{0}+0.5 h d_{0}^{j} y_{0}^{(\sigma)}\right)\right. \\
&+\left(\bar{x}_{N}^{m}-x_{N}^{m}\right) \bar{y}_{N}\left(\tilde{\mu}_{2}-\tilde{\beta}_{1} y_{N}^{(\sigma)}-\tilde{\beta}_{2} \Delta_{0 t_{j+\sigma}}^{\alpha} y_{N}\right)+x_{N}^{m} \tilde{\mu}_{2} \bar{y}_{N} \\
&= x_{0.5}^{m} y_{0}^{(\sigma)} \tilde{\mu}_{1}+x_{0.5}^{m} \tilde{\mu}_{1} \Delta_{0 t_{j+\sigma}}^{\alpha} y_{0}-\frac{0.5 h}{m+1} x_{0.5}^{m} y_{0}^{(\sigma)} \Delta_{0 t_{j+\sigma}}^{\alpha} y_{0} \\
&-\frac{0.5 h}{m+1} x_{0.5}^{m}\left(\Delta_{0 t_{j+\sigma}}^{\alpha} y_{0}\right)^{2}-\frac{0.25 h^{2}}{m+1} x_{0.5}^{m} y_{0}^{(\sigma)} d_{0}^{j} y_{0}^{(\sigma)} \\
&-\frac{0.25 h^{2}}{m+1} x_{0.5}^{m} d_{0}^{j} y_{0}^{(\sigma)} \Delta_{0 t_{j+\sigma}}^{\alpha} y_{0}+\bar{x}_{N}^{m} y_{N}^{(\sigma)} \tilde{\mu}_{2}+\bar{x}_{N}^{m} \tilde{\mu}_{2} \Delta_{0 t_{j+\sigma}}^{\alpha} y_{N} \\
&-\left(\bar{x}_{N}^{m}-x_{N}^{m}\right) \tilde{\beta}_{1}\left(y_{N}^{\sigma}\right)^{2}-\left(\bar{x}_{N}^{m}-x_{N}^{m}\right) \tilde{\beta}_{1} y_{N}^{(\sigma)} \Delta_{0 t_{j+\sigma}}^{\alpha} y_{N} \\
&-\left(\bar{x}_{N}^{m}-x_{N}^{m}\right) y_{N}^{(\sigma)} \tilde{\beta}_{2} \Delta_{0 t_{j+\sigma}}^{\alpha} y_{N}-\left(\bar{x}_{N}^{m}-x_{N}^{m}\right) \tilde{\beta}_{2}\left(\Delta_{0 t_{j+\sigma}}^{\alpha} y_{N}\right)^{2} \\
& \leqslant \varepsilon_{3}\left(\Delta_{0 t_{j+\sigma}}^{\alpha} y_{0}\right)^{2}+\varepsilon_{4}\left(\Delta_{0 t_{j+\sigma}}^{\alpha} y_{N}\right)^{2}+M_{12}^{\varepsilon_{3}, \varepsilon_{4}}\left(\tilde{\mu}_{1}^{2}+\tilde{\mu}_{2}^{2}\right) \\
&\left.\left.+M_{13}^{\varepsilon_{3}, \varepsilon_{4}}\left(\| x^{\frac{m}{2}} y^{(\sigma)}\right]_{0}^{2}+\left(x_{0.5}^{\frac{m}{2}} y_{0}\right)^{2}+\| \bar{x}^{\frac{m}{2}} y_{\bar{x}}^{(\sigma)}\right]\left.\right|_{0} ^{2}\right) \\
&-\frac{h}{4(m+1)} x_{0.5}^{m} \Delta_{0 t_{j+\sigma}}^{\alpha} y_{0}^{2}-\left(\bar{x}_{N}^{m}-x_{N}^{m}\right) \frac{\tilde{\beta}_{2}}{2} \Delta_{0 t_{j+\sigma}}^{\alpha}\left(y_{N}\right)^{2} \\
&-\frac{0.5 h}{m+1} x_{0.5}^{m}\left(\Delta_{0 t_{j+\sigma}}^{\alpha} y_{0}\right)^{2}-\left(\bar{x}_{N}^{m}-x_{N}^{m}\right) \tilde{\beta}_{2}\left(\Delta_{0 t_{j+\sigma}}^{\alpha} y_{N}\right)^{2} . \\
&
\end{aligned}
$$

In view of above identities, for

$$
\varepsilon_{1}=\frac{\overline{\bar{\varkappa}}}{2}, \quad \varepsilon_{2}=\frac{\beta_{2} x_{N}^{m}}{2}, \quad \varepsilon_{3}=\frac{h x_{0.5}^{m}}{4(m+1)}, \quad \varepsilon_{4}=\left(\bar{x}_{N}^{m}-x_{N}^{m}\right) \frac{\tilde{\beta}_{2}}{2}
$$

by (6.19) we find:

$$
\begin{aligned}
\left(\frac{\overline{\bar{\varkappa}}}{2}, \Delta_{0 t_{j+\sigma}}^{\alpha}\left(x^{\frac{m}{2}} y\right)^{2}\right] & \left.\left.+M_{10} \Delta_{0 t_{j+\sigma}}^{\alpha} \| \bar{x}^{\frac{m}{2}} y_{\bar{x}}\right]\left.\right|_{0} ^{2}+M_{3} \| \bar{x}^{\frac{m}{2}} y_{\bar{x}}^{(\sigma)}\right]\left.\right|_{0} ^{2}+\left(\frac{\overline{\bar{\varkappa}}}{2},\left(\Delta_{0 t_{j+\sigma}}^{\alpha}\left(x^{\frac{m}{2}} y\right)\right)^{2}\right] \\
& \left.+c_{0} \| \Delta_{0 t_{j+\sigma}}^{\alpha} \bar{x}^{\frac{m}{2}} y_{\bar{x}}\right]\left.\right|_{0} ^{2}+\frac{h}{4(m+1)} x_{0.5}^{m} \Delta_{0 t_{j+\sigma}}^{\alpha} y_{0}^{2} \\
& +\left(\frac{\tilde{\varkappa} \beta_{2}}{2} x_{N}^{m}+\left(\bar{x}_{N}^{m}-x_{N}^{m}\right) \frac{\tilde{\beta}_{2}}{2}\right) \Delta_{0 t_{j+\sigma}}^{\alpha}\left(y_{N}\right)^{2} \\
& +\frac{0.5 h}{2(m+1)} x_{0.5}^{m}\left(\Delta_{0 t_{j+\sigma}}^{\alpha} y_{0}\right)^{2} \\
& +\left(\frac{\tilde{\varkappa} \beta_{2}}{2} x_{N}^{m}+\left(\bar{x}_{N}^{m}-x_{N}^{m}\right) \frac{\tilde{\beta}_{2}}{2}\right)\left(\Delta_{0 t_{j+\sigma}}^{\alpha} y_{N}\right)^{2} \\
\leqslant & M_{14}\left\|x^{\frac{m}{2}} \varphi\right\|_{0}^{2}+M_{15}\left(\tilde{\mu}_{1}^{2}+\tilde{\mu}_{2}^{2}\right) \\
& \left.\left.+\left.M_{16}\left(\| x^{\frac{m}{2}} y^{\sigma}\right]\right|_{0} ^{2}+\| \bar{x}^{\frac{m}{2}} y_{\bar{x}}^{(\sigma)}\right]\left.\right|_{0} ^{2}+\left(x_{0.5}^{\frac{m}{2}} y_{0}\right)^{2}\right) .
\end{aligned}
$$


Now we transform the first, fourth, seventh and ninth terms in the left hand side in (6.21) bearing in mind the inequality $x_{N-0.5}^{m} \geqslant \frac{1}{6} x_{N}^{m}$ :

$$
\begin{aligned}
& \left(\frac{\overline{\bar{\varkappa}}}{2}, \Delta_{0 t_{j+\sigma}}^{\alpha}\left(x^{\frac{m}{2}} y\right)^{2}\right]+\left(\frac{\tilde{\varkappa} \beta_{2}}{2} x_{N}^{m}+\left(\bar{x}_{N}^{m}-x_{N}^{m}\right) \frac{\tilde{\beta}_{2}}{2}\right) \Delta_{0 t_{j+\sigma}}^{\alpha}\left(y_{N}\right)^{2}+\left(\frac{\overline{\bar{\varkappa}}}{2},\left(\Delta_{0 t_{j+\sigma}}^{\alpha}\left(x^{\frac{m}{2}} y\right)\right)^{2}\right] \\
& +\left(\frac{\tilde{\varkappa} \beta_{2}}{2} x_{N}^{m}+\left(\bar{x}_{N}^{m}-x_{N}^{m}\right) \frac{\tilde{\beta}_{2}}{2}\right)\left(\Delta_{0 t_{j+\sigma}}^{\alpha} y_{N}\right)^{2} \\
& =\left(\frac{\bar{\varkappa}}{2}, \Delta_{0 t_{j+\sigma}}^{\alpha}\left(x^{\frac{m}{2}} y\right)^{2}\right)+\frac{0.5 h}{2} x_{N}^{m} \Delta_{0 t_{j+\sigma}}^{\alpha}\left(y_{N}\right)^{2}+\left(\frac{\bar{\varkappa}}{2},\left(\Delta_{0 t_{j+\sigma}}^{\alpha}\left(x^{\frac{m}{2}} y\right)\right)^{2}\right) \\
& +\frac{0.5 h}{2} x_{N}^{m}\left(\Delta_{0 t_{j+\sigma}}^{\alpha} y_{N}\right)^{2}+\left(\frac{\tilde{\varkappa} \beta_{2}}{2} x_{N}^{m}+\left(\bar{x}_{N}^{m}-x_{N}^{m}\right) \frac{\tilde{\beta}_{2}}{2}\right) \Delta_{0 t_{j+\sigma}}^{\alpha}\left(y_{N}\right)^{2} \\
& +\left(\frac{\tilde{\varkappa} \beta_{2}}{2} x_{N}^{m}+\left(\bar{x}_{N}^{m}-x_{N}^{m}\right) \frac{\tilde{\beta}_{2}}{2}\right)\left(\Delta_{0 t_{j+\sigma}}^{\alpha} y_{N}\right)^{2} \\
& =\left(\frac{\bar{\varkappa}}{2}, \Delta_{0 t_{j+\sigma}}^{\alpha}\left(x^{\frac{m}{2}} y\right)^{2}\right)+\left(\frac{\bar{\varkappa}}{2},\left(\Delta_{0 t_{j+\sigma}}^{\alpha}\left(x^{\frac{m}{2}} y\right)\right)^{2}\right) \\
& +\left(\frac{\tilde{\varkappa} \beta_{2}}{2} \bar{x}_{N}^{m}+\frac{0.5 h}{2} \bar{x}_{N}^{m}\right) \Delta_{0 t_{j+\sigma}}^{\alpha}\left(y_{N}\right)^{2} \\
& +\left(\frac{\tilde{\varkappa} \beta_{2}}{2} \bar{x}_{N}^{m}+\frac{0.5 h}{2} \bar{x}_{N}^{m}\right)\left(\Delta_{0 t_{j+\sigma}}^{\alpha} y_{N}\right)^{2} \\
& \geqslant \frac{M_{17}}{2}\left(1, \Delta_{0 t_{j+\sigma}}^{\alpha}\left(x^{\frac{m}{2}} y\right)^{2}\right)+\frac{h}{4} \bar{x}_{N}^{m} \Delta_{0 t_{j+\sigma}}^{\alpha}\left(y_{N}\right)^{2} \\
& +\frac{M_{17}}{2}\left(1,\left(\Delta_{0 t_{j+\sigma}}^{\alpha}\left(x^{\frac{m}{2}} y\right)\right)^{2}\right)+\frac{h}{4} \bar{x}_{N}^{m}\left(\Delta_{0 t_{j+\sigma}}^{\alpha} y_{N}\right)^{2} \\
& \geqslant \frac{1}{4}\left(1, \Delta_{0 t_{j+\sigma}}^{\alpha}\left(x^{\frac{m}{2}} y\right)^{2}\right)+\frac{0.5 h}{12} x_{N}^{m} \Delta_{0 t_{j+\sigma}}^{\alpha}\left(y_{N}\right)^{2} \\
& +\frac{1}{4}\left(1,\left(\Delta_{0 t_{j+\sigma}}^{\alpha}\left(x^{\frac{m}{2}} y\right)\right)^{2}\right)+\frac{0.5 h}{12} x_{N}^{m}\left(\Delta_{0 t_{j+\sigma}}^{\alpha} y_{N}\right)^{2} \\
& \left.\left.\geqslant \frac{1}{12} \Delta_{0 t_{j+\sigma}}^{\alpha} \| x^{\frac{m}{2}} y\right]\left.\right|_{0} ^{2}+\frac{1}{12} \| \Delta_{0 t_{j+\sigma}}^{\alpha} x^{\frac{m}{2}} y\right]\left.\right|_{0} ^{2},
\end{aligned}
$$

where

$$
M_{17}=\left\{\begin{array}{lll}
1 \quad \text { as } \quad m=0 \quad \text { or } & m \geqslant 1 \\
\frac{1}{2} \quad \text { as } & m \in(0,1), & h \leqslant h_{0}=\sqrt{\frac{12 x^{2}}{m(1-m)}} .
\end{array}\right.
$$

By (6.22) and (6.21) we obtain:

$$
\begin{gathered}
\left.\left.\left.\left.\Delta_{0 t_{j+\sigma}}^{\alpha} \| x^{\frac{m}{2}} y\right]\left.\right|_{1} ^{2}+\| \bar{x}^{\frac{m}{2}} y_{\bar{x}}^{(\sigma)}\right]\left.\right|_{0} ^{2}+\| \Delta_{0 t_{j+\sigma}}^{\alpha} x^{\frac{m}{2}} y\right]\left.\right|_{0} ^{2}+\| \Delta_{0 t_{j+\sigma}}^{\alpha} \bar{x}^{\frac{m}{2}} y_{\bar{x}}\right]\left.\right|_{0} ^{2} \\
\left.\leqslant M_{18} \| x^{\frac{m}{2}} y^{(\sigma)}\right]\left.\right|_{1} ^{2}+M_{19}\left(\left\|x^{\frac{m}{2}} \varphi\right\|_{0}^{2}+\tilde{\mu}_{1}^{2}+\tilde{\mu}_{2}^{2}\right),
\end{gathered}
$$

where

$$
\left.\left.\| x^{\frac{m}{2}} y\right]\left.\right|_{1} ^{2}=\| x^{\frac{m}{2}} y\right]\left.\right|_{0} ^{2}+\left\|x^{\frac{m}{2}} y_{\bar{x}}\right\|_{0}^{2}+\left(x_{0.5}^{\frac{m}{2}} y_{0}\right)^{2} .
$$

Reproducing the arguing in (3.18)-(3.27), by (6.23) we find the sought apriori estimate:

$$
\left.\left.\| x^{\frac{m}{2}} y^{j+1}\right]\left.\right|_{1} ^{2} \leqslant\left. M\left(\| x^{\frac{m}{2}} y^{0}\right]\right|_{1} ^{2}+\frac{t_{j}^{\alpha}}{\Gamma(1+\alpha)} \max _{0 \leqslant j^{\prime} \leqslant j}\left(\left\|x^{\frac{m}{2}} \varphi\right\|_{0}^{2}+\tilde{\mu}_{1}^{2}+\tilde{\mu}_{2}^{2}\right)\right),
$$


where $M$ is a positive constant independent of $h$ and $\tau$.

Theorem 4. Assume that conditions (1.4), (4.5) hold. Then there exits $\tau_{0}, h_{0}$ such that as $\tau \leqslant \tau_{0}, h \leqslant h_{0}$, the solution of difference problem (6.1)-(6.4) satisfies apriori estimate (6.24).

Apriori estimate (6.24) imply the uniqueness of the solution of problem (6.1)-(6.4) and its stability with respect to the initial data and the right hand side.

Let $u(x, t)$ be the solution of problem (4.1)-(4.4), $y\left(x_{i}, t_{j}\right)=y_{i}^{j}$ be the solution of difference problem (6.1)-(6.4). To estimate the exactness of difference scheme (6.1)-(6.4), we consider the difference $z_{i}^{j}=y_{i}^{j}-u_{i}^{j}$, where $u_{i}^{j}=u\left(x_{i}, t_{j}\right)$. Then substituting $y=z+u$ into (6.1)-(6.4), we obtain the problem for the function $z$ :

$$
\begin{gathered}
\bar{\varkappa}_{0 t_{j+\sigma}}^{\alpha} z=\frac{\varkappa}{x_{i}^{m}}\left(x_{i-0.5}^{m} a^{j} z_{\bar{x}}^{(\sigma)}\right)_{x}+\frac{1}{x_{i}^{m}} \Delta_{0 t_{j+\sigma}}^{\alpha}\left(x_{i-0.5}^{m} \gamma_{i} z_{\bar{x}, i}\right)_{x}+\frac{b^{-j}}{x_{i}^{m}}\left(x_{i-0.5}^{m} a_{i}^{j} z_{\bar{x}, i}^{(\sigma)}\right) \\
+\frac{b^{+j}}{x_{i}^{m}}\left(x_{i+0.5}^{m} a_{i+1}^{j} z_{x, i}^{(\sigma)}\right)-\sum_{s=0}^{i} d_{s}^{j} z_{s}^{(\sigma)} \hbar+\Psi_{i}^{j}, \quad(x, t) \in \omega_{h, \tau}, \\
\varkappa_{0} a_{1} z_{x, 0}^{(\sigma)}+\Delta_{0 t_{j+\sigma}}^{\alpha}\left(\gamma_{1} z_{\bar{x}, 0}\right)=\frac{0.5 h}{m+1}\left(\Delta_{0 t_{j+\sigma}}^{\alpha} z_{0}+0.5 h d_{0}^{j} z_{0}^{(\sigma)}\right)-\tilde{\nu}_{1}, \\
-\varkappa_{N} a_{N} z_{\bar{x}, N}^{(\sigma)}-\Delta_{0 t_{j+\sigma}}^{\alpha}\left(\gamma_{N} z_{\bar{x}, N}\right)=\tilde{\beta}_{1} z_{N}^{(\sigma)}+0.5 h \sum_{s=0}^{N} d_{s}^{j} z_{s}^{(\sigma)} \hbar+\tilde{\beta}_{2} \Delta_{0 t_{j+\sigma}}^{\alpha} z_{N}-\tilde{\nu}_{2}, \\
z(x, 0)=0, \quad x \in \bar{\omega}_{h},
\end{gathered}
$$

where $\|x \Psi\|_{0}^{2}=O\left(h^{2}+\tau^{2}\right), \tilde{\nu}_{1}=O\left(h^{2}+\tau^{2}\right), \tilde{\nu}_{2}=O\left(h^{2}+\tau^{2}\right)$ are the errors of approximation differential problem (4.1)-(4.4) by difference scheme (6.1)-(6.4) in the class of solutions $u=$ $u(x, t)$ of problem $(4.1)-(4.4)$, see [25].

Applying apriori estimate (6.24) for solution of problem (6.25)-(6.28), we obtain the inequality

$$
\left.\| x^{\frac{m}{2}} z^{j+1}\right]\left.\right|_{1} ^{2} \leqslant M \max _{0 \leqslant j^{\prime} \leqslant j}\left(\left\|x^{\frac{m}{2}} \Psi^{j^{\prime}}\right\|_{0}^{2}+\nu_{1}^{2}+\nu_{2}^{2}\right)
$$

where $M$ is a positive constant independent of $h$ and $\tau$. This implies the apriori estimate

$$
\left.\| x z^{j+1}\right]\left.\right|_{1} ^{2} \leqslant \bar{M} \max _{0 \leqslant j^{\prime} \leqslant j}\left(\left\|x \Psi^{j^{\prime}}\right\|_{0}^{2}+\nu_{1}^{2}+\nu_{2}^{2}\right)
$$

where $\bar{M}$ is a positive constant independent of $h$ and $\tau$. Apriori estimate (6.30) implies the convergence of the solution of difference problem (5.1)-(5.4) to the solution of differential problem (1.1), (1.2), (4.1), (1.4) in the sense of the norm $\left.\| x z^{j+1}\right]\left.\right|_{1} ^{2}$ on each fiber so that there exist $\tau_{0}, h_{0}$ such that as $\tau \leqslant \tau_{0}, h \leqslant h_{0}$, the apriori estimate holds:

$$
\left.\| x\left(y^{j+1}-u^{j+1}\right)\right]\left.\right|_{1} \leqslant \bar{M}\left(h^{2}+\tau^{2}\right) .
$$

Corollary 2. The obtained results hold true also in the case, when equation (4.1) is of the form:

$$
\begin{aligned}
\partial_{0 t}^{\alpha} u= & \frac{1}{x^{m}} \frac{\partial}{\partial x}\left(x^{m} k(x, t) \frac{\partial u}{\partial x}\right)+\frac{1}{x^{m}} \partial_{0 t}^{\alpha} \frac{\partial}{\partial x}\left(x^{m} \eta(x) \frac{\partial u}{\partial x}\right) \\
& +r(x, t) \frac{\partial u}{\partial x}-\int_{0}^{l} q(x, t) u(x, t) d x+f(x, t), \quad 0<x<l, \quad 0<t \leqslant T,
\end{aligned}
$$

if we assume the inequality $|q| \leqslant c_{2}$. 


\section{BIBLIOGRAPHY}

1. A.M. Nakhushev. Fractional calculus and its application. Fizmatgiz, Moscow (2003). (in Russian).

2. V.V. Uchaikin. Method of fractional derivatives. Artishok Publ., Ulyanovsk (2008). (in Russian).

3. B.B. Mandelbrot. The Fractal geometry of nature. W.H. Freeman and Company, New York (1982).

4. R.L. Begli, P.J. Torvik. Differential calculus based on fractional order derivatives: a new approach for calculating construction with viscoelastic damping // Aerokosmich. Tekh. 2:2, 84-93 (1984). (in Russian).

5. J. Feder. Fractals. Plenum Press, New York (1988).

6. O.Yu. Dinariev. Flow in a fractured medium with fractal fracture geometry // Izv. Akad. Nauk SSSR, Mekh. Zhidk. Gaza. 25:5, 66-70 (1990). [Fluid Dyn. 25:5, 704-708 (1990).]

7. R.R. Nigmatullin. The realization of generalized transfer equation in a medium with fractal geometry // Phys. Status Solidi. B. 133:1, 425-430 (1986).

8. A.N. Kochubei. Fractional-order diffusion // Differ. Uravn. 26:4, 660-670 (1990). [Differ. Equat. 26:4, 485-492 (1990).]

9. K.V. Chukbar. Stochastic transport and fractional derivatives // Zhurn. Exper. Teor. Fiz. 108:5, 1875-1884 (1995). [J. Exp. Theor. Phys. 81:5, 1025-1029 (1995).]

10. G.I. Barenblatt, Yu.P. Zheltov, I.N. Kochina. Basic Concepts in the theory of seepage of homogeneous liquids in fissured rocks (strata) // Prikl. Matem. Mekh. 24:5, 852-864 (1960). [J. Appl. Math. Mekh. 24:5, 1286-1303 (1960).]

11. E.S. Dzektser. Equations of motion of underground water with a free surface in stratified media // DAN SSSR. 220:3, 540-543 (1975). (in Russian).

12. M. Hallaire. Effective water potential in soil drying. Gidrometeoizdat, Leningrad (1966). [Le potential efficace de l'eau dans le sol en régime de dessèchement // L'eau et production vegetale. I.N.R.A., 27-62 (1964).]

13. S.V. Nerpin, A.F. Chudnovsky. Energy- and mass-transfer in a system plant-soil-air. Gidrometeoizdat, Leningrad (1975). (in Russian).

14. P.J. Chen, M.E. Curtin. On a theory of heat conduction involving two temperatures // J. Angew. Math. Phys. 19:4, 614-627 (1968).

15. R.E. Showalter, T.W. Ting. Pseudoparabolic partial differential equations // SIAM J. Math. Anal. 1:1, 1-26 (1970).

16. S.L. Sobolev. On a new problem of mathematical physics // Izv. AN SSSR. Ser. Matem. 18:1, 3-50 (1954). (in Russian).

17. A.G. Sveshnikov, A.B. Al'shin, M.O. Korpusov, Yu.D. Pletner. Linear and nonlinear equations of Sobolev type. Moscow, Fizmatlit (2007). (in Russian).

18. M.Kh. Shkhanukov. Some boundary-value problems for a third-order equation, and extremal properties of its solutions // Differ. Uravn. 19:1, 145-152 (1983). [Differ. Equat. 19:1, 124-130 (1983).]

19. V.A. Vodakhova. On a certain boundary value problem for a third order equation with a non-local condition of A. M. Nakhushev // Differ. Uravn. 19:1, 163-166 (1983). (in Russian).

20. M.Kh. Beshtokov. On the numerical solution of a nonlocal boundary value problem for a degenerating pseudoparabolic equation // Diff. Uravn. 52:10, 1393-1406 (2016). [Diff. Equat. 52:10, 1341-1354 (2016).]

21. M.Kh. Beshtokov. Difference method for solving a nonlocal boundary value problem for a degenerating third-order pseudo-parabolic equation with variable coefficients // Zhurn. Vychisl. Matem. Matem. Fiz. 56:10, 1780-1794 (2016). [Comp. Math. Math. Phys. 56:10, 1763-1777 (2016).]

22. M.Kh. Beshtokov. On a non-local boundary value problem for the third order pseudo-parabolic equation // Comp. Math. Model. 27:1, 60-79 (2016).

23. M.Kh. Beshtokov. The third boundary value problem for loaded differential Sobolev type equation and grid methods of their numerical implementation // IOP Conf. Ser: Materials Science and Engineering. 158:1, id 012019 (2016).

24. M.Kh. Beshtokov. Differential and difference boundary value problem for loaded third-order pseudoparabolic differential equations and difference methods for their numerical solution // Zhurn. Vychisl. Matem. Matem. Fiz. 57:12, 2021-2041 (2017). [Comp. Math. Math. Phys. 57:12, 1973-1993 (2017).] 
25. M.Kh. Beshtokov. Local and nonlocal boundary value problems for degenerating and nondegenerating pseudoparabolic equations with a Riemann-Liouville fractional derivative // Diff. Uravn. 54:6, 763-778 (2018). [Diff. Equat. 54:6, 758-774 (2018).]

26. H. Caputo. Lineal model of dissipation whose $Q$ is almost frequency independent-II // Geophys J. Astronom. Soc. 13:5, 529-539 (1967).

27. A.N. Gerasimov. Generalization of linear deformation laws and their application to problems of internal friction // Prikl. Matem. Mekh. 12, 251-260 (1948). (in Russian).

28. A.A. Alikhanov. A priori estimates for solutions of boundary value problems for fractional-order equations // Diff. Uravn. 46:5, 658-664 (2010). [Diff. Equat. 46:5, 660-666 (2010).]

29. A.A. Samarski. The theory of difference schemes. Nauka, Moscow, 1989. [Marcel Dekker, New York (2001).]

30. A.A. Alikhanov. A new difference scheme for the time fractional diffusion equation // J. Comp. Phys. 280:1, 424-438 (2015).

31. D. Li, H.-L. Liao, W. Sun, J. Wang and J. Zhang. Analysis of $L_{1}$-Galerkin FEMs for time-fractional nonlinear parabolic problems // Comm. Comp. Phys. 24:86, 86-103 (2018).

Murat Khamidbievich Beshtokov,

Institute of Applied Mathematics and Automatization,

Kabardino-Balkar Scientific Center RAS,

Shortanova, 89A,

360000, Nalchik, Russia

E-mail: beshtokov-murat@yandex.ru 